\title{
Colour distributions in E-S0 galaxies.
}

\author{
V. Colour data for strongly inclined lenticulars ${ }^{\star}$ \\ R. Michard ${ }^{1,2}$ and P. Poulain ${ }^{3}$ \\ 1 Observatoire de la Côte d'Azur, Dept. Augustin Fresnel, BP. 4229, F-06304 Nice Cedex 4, France \\ 2 Observatoire de Paris, DEMIRM, 77 avenue Denfert-Rochereau, F-75015 Paris, France \\ ${ }^{3}$ Observatoire Midi-Pyrénées, 14 avenue Edouard Bélin, F-31400 Toulouse, France
}

Received May 12; accepted September 29, 1999

\begin{abstract}
Colours distributions have been studied in 12 S0 galaxies, most of these nearly edge-on, from high resolution frames obtained at the CFHT and the Pic du Midi $2 \mathrm{~m}$ telescope, supplemented for some purposes by low resolution frames from the Observatoire de Haute Provence $120 \mathrm{~cm}$ telescope.
\end{abstract}

Great attention has been given to reduce the effects of "differential seeing", resulting from different PSF's in the two frames needed for a colour measurement. Errors from various sources, and their effect upon measured quantities, have been evaluated from the study of pseudo-colours (obtained by applying our measuring techniques to pair of frames taken in the same passband), and from the comparison of colours data from our various series.

Data are given about the following topics:

- Radial isophotal colour profiles, leading to reference colours at $r_{\mathrm{e}}$ and gradients in 4 colour indices (except for missing data);

- Reference colours and gradients in possibly dustless regions of nearly pure bulge or disk, allowing a comparison between the two components;

- Dust patterns, seen in 7 objects;

- Systematic differences between major and minor axis colours upon isophotal contours (outside dust patterns). Both a redder or bluer major axis are encountered depending upon the object, or the central distance in a given object. The observations may sometimes be explained by local dust concentration in disks. In other cases population differences between spheroidal and disk components should be invoked;

- Asymmetries in light and colour distributions along the minor axis in 3 objects, due to dust concentrated in the disk;

Send offprint requests to: R. Michard

* Based on observations collected at the Canada-FranceHawaii Telescope, the Observatoire du Pic du Midi and the Observatoire de Haute-Provence.
- Correlations between ring structure and major axis colour profiles.

Key words: galaxies: elliptical and lenticulars, CD galaxies: ISM

\section{Introduction}

\subsection{Background of this contribution}

The colour distributions in S0-galaxies have not been much studied, although they are expected to be more complex than happens for pure spheroidal objects. Indeed Sandage (1961) introduced the three subclasses $\mathrm{S}_{1}, \mathrm{~S}_{2}$, and $\mathrm{S}_{3}$, to represent three degrees in the visibility of dust, from none seen to obvious. It may be also expected that the two main components of any S0, i.e. the bulge and the disk (and perhaps also the envelope), will present different stellar populations, assuming some analogy with galaxies of later types. Pursuing the analogy, we might predict bluer populations, but perhaps also more dust in the disk, leading to rather complex colour distributions.

A study of NGC 3115 by Silva et al. (1989) points to a disk much bluer than the bulge. Bothun \& Gregg (1990) studied a significant sample of S0's by $B-H$ and $H-K$ photometry, and found "evidence for star formation 5 gigayears ago" in a number of disks. Some papers dealing with both S0's and disk galaxies of later types are obviously relevant. Balcells \& Peletier (1994) (BP94) studied the colour profiles in bulges. The same authors (Peletier \& Balcells 1996), compared bulge and disk colours from $U B R J K$ photometry for a large sample of S0-Sbc objects. They find some tendency for the disks to be bluer, but with a large scatter: this is not surprising in view of the mixed Hubble types in the sample, and of the necessarily 
coarse definition of bulge and disk colours respectively. A follow-up of this work is the survey by Peletier \& Balcells (1997).

Some years ago a program was started to obtain some data on the colour distributions in S0's, using good resolution frames from the Pic du Midi, plus a few from the CFHT. This program was not favoured with good weather and usable data are available only for a dozen objects of high inclination to the line of sight. In some cases the high resolution frames could be supplemented with frames from the Observatoire de Haute-Provence, of much lesser resolution, but larger field and often better $\mathrm{S} / \mathrm{N}$. The OHP observations were previously used in morphological studies of E-S0 galaxies, including the present sample, by Michard \& Marchal (1993) (or MM93) and (1994) (or MM94).

This paper presents the data from this small sample. Section 2 reviews the observations, Sect. 3 the data analysis: as in previous papers of this series, it has been tried to reduce the errors due to "differential seeing", that is the different PSF of the two frames needed for measuring a colour. Section 4 collects the data mostly in tabular form, with colour maps and graphs of radial colour profiles assembled in the Appendix. Section 5 gives some tentative conclusions.

\subsection{Notations and abbreviations}

It may be useful to collect here the notations and abbreviations currently used below.

. SuBr surface brightness;

. majA, minA, major and minor axis respectively;

. $a, c$ major and minor axis of the Reference Ellipse for the representation of an isophote according to Carter; . $r=(a c)^{1 / 2}$ mean radius of the isophote;

. $l, s$, true major and minor axis respecticely;

. $e_{i}, f_{i}$ coefficients of cosine and sine terms in Carter's harmonic representation of deviations from the Reference Ellipse;

. PA position angle of major axis;

- $q$ axis ratio of the Reference Ellipse;

. $\epsilon=1-q$ ellipticity;

. spH spheroidal halo; thD thick disk; exD extended disk, are symbols of our classification of the envelopes of disky galaxies (see MM94).

\section{Observations}

The observations used in this work have been gathered at three different instruments:

1. High resolution frames were obtained for 5 objects at the CFHT, in 1989-90 by J.L. Nieto and 1992 by one of us. The so called HRCam was used, with a scale of $0.107 \mathrm{arcsec} /$ pixel. The total field was only $641 \times$
Table 1. Literature data for sample galaxies. Types are from the RSA. The velocities $V_{\text {op }}$ and galactic $B$ absorption $A_{\mathrm{g}}$ are from the RC3. The total magnitudes $V_{\mathrm{T}}$, the radius $\log r_{\mathrm{e}}$ in arcsec, the estimated luminosity $M_{\mathrm{T}}$ in $B$ are from MM94. Env. gives our classification of the outer envelopes (the notations are explained above in I.2)

\begin{tabular}{llllllll}
\hline NGC & Type & $V_{\mathrm{opt}}$ & $A_{\mathrm{g}}$ & $V_{\mathrm{T}}$ & $\log r_{\mathrm{e}}$ & $-M_{\mathrm{T}}$ & Env. \\
\hline 2549 & $\mathrm{~S}_{1}(7)$ & 1070 & 0.13 & 11.20 & 1.05 & 19.12 & $\mathrm{exD}$ \\
3098 & $\mathrm{~S}_{1}(9)$ & 1401 & 0.11 & 12.06 & 0.90 & 18.43 & $\mathrm{spH}$ \\
3115 & $\mathrm{~S}_{1}(7)$ & 670 & 0.10 & 9.00 & 1.61 & 19.91 & $\mathrm{spH}$ \\
3245 & $\mathrm{~S}_{1}(5)$ & 1348 & 0.05 & 10.90 & 1.32 & 19.05 & $\mathrm{thD}$ \\
4026 & $\mathrm{~S}_{1}(9)$ & 930 & 0.04 & 10.53 & 1.21 & 19.44 & thD \\
4036 & $\mathrm{~S}_{3}(8)$ & 1397 & 0.03 & 10.69 & 1.30 & 19.33 & $\mathrm{exD}$ \\
4111 & $\mathrm{~S}_{1}(9)$ & 815 & 0.00 & 10.63 & 1.02 & 19.01 & thD \\
4350 & $\mathrm{~S}_{1}(8)$ & 1234 & 0.03 & 11.00 & 1.16 & 19.11 & $\mathrm{spH}$ \\
5308 & $\mathrm{~S}_{1}(8)$ & 2041 & 0.01 & 11.47 & 1.10 & 18.96 & thD \\
5422 & $\mathrm{~S}_{3}(8)$ & 1782 & 0.03 & 11.92 & 1.12 & 19.05 & thD \\
7332 & $\mathrm{~S}_{2}(8)$ & 1202 & 0.12 & 11.00 & 1.13 & 18.62 & thD \\
7457 & $\mathrm{~S}_{1}(5)$ & 824 & 0.22 & 11.14 & 1.52 & 18.86 & $\mathrm{exD}$ \\
\hline
\end{tabular}

Table 2. First list of frames used in this work. OBS = Telescope, coded as CFH, TBL for the $2 \mathrm{~m}$ Bernard Lyot telescope at the Pic du Midi, OHP for the $120 \mathrm{~cm}$ telescope at Observatoire de Haute-Provence. $F=$ filter. Exposure times are in seconds. For the CFHT, dates are for the begining of the night in HST; for the TBL or OHP they are for the begining of the night in UT. $W_{0}=F W H M$ in arcsec of the original PSF. $W_{\mathrm{i}}=$ same for the "improved" PSF (see Sect. 3). When the file code contains an $\mathrm{m}$, it was obtained by merging two frames

\begin{tabular}{|c|c|c|c|c|c|c|c|}
\hline $\mathrm{NGC}$ & OBS & $F$ & File & Date & Exp. & $\overline{W_{0}}$ & $W_{\mathrm{i}}$ \\
\hline 2549 & $\mathrm{CFH}$ & $\bar{B}$ & $\mathrm{~h} 042$ & $04 / 04 / 92$ & 900 & 0.59 & - \\
\hline id & id & $R$ & h042 & id & 60 & 0.62 & - \\
\hline id & $\mathrm{OHP}$ & $B$ & $2 \mathrm{~b} 33$ & $18 / 01 / 91$ & 1080 & 2.66 & 1.98 \\
\hline id & id & $V$ & $2 \mathrm{~b} 30$ & id & 180 & 2.26 & 2.00 \\
\hline id & id & $R$ & 2b31 & id & 120 & 2.10 & 1.94 \\
\hline id & id & $I$ & 2b32 & id & 120 & 2.39 & 1.82 \\
\hline id & id & $U$ & $6 a 17$ & $13 / 01 / 94$ & 3000 & 2.92 & 2.00 \\
\hline id & id & $V$ & $6 a 16$ & id & 150 & 2.52 & 1.85 \\
\hline 3098 & $\mathrm{CFH}$ & $B$ & h032 & 03/04/92 & 900 & 0.54 & - \\
\hline id & id & $R$ & h033 & id & 60 & 0.61 & - \\
\hline id & $\mathrm{OHP}$ & $B$ & $2 \mathrm{~b} 48$ & $18 / 01 / 91$ & 1200 & 2.96 & 2.60 \\
\hline id & id & $V$ & $2 \mathrm{~b} 45$ & id & 360 & 2.54 & - \\
\hline id & id & $R$ & $2 \mathrm{~b} 46$ & id & 300 & 2.91 & 2.60 \\
\hline id & id & $I$ & $2 \mathrm{~b} 47$ & id & 300 & 2.88 & 2.47 \\
\hline id & id & $U$ & $6 a 61$ & $14 / 01 / 94$ & 3600 & 4.93 & 3.75 \\
\hline id & id & $U$ & $6 a 62$ & id & 3600 & 4.90 & 3.75 \\
\hline id & id & $V$ & $6 a 60$ & id & 120 & 4.29 & 3.75 \\
\hline 3115 & $\mathrm{CFH}$ & $B$ & h06m & 06/12/89 & 90 & 0.73 & - \\
\hline id & id & $R$ & h064 & id & 60 & 0.55 & - \\
\hline id & OHP & $U$ & $3 c 33$ & $18 / 03 / 91$ & 2700 & 3.85 & 3.00 \\
\hline id & id & $B$ & $3 c 34$ & id & 600 & 3.06 & 2.75 \\
\hline id & id & $V$ & $3 \mathrm{~b} 28$ & $16 / 03 / 91$ & 180 & 3.00 & 2.51 \\
\hline id & id & $R$ & $3 c 32$ & $18 / 03 / 91$ & 180 & 2.70 & - \\
\hline id & id & $I$ & $3 c 35$ & id & 300 & 2.50 & - \\
\hline 3245 & $\mathrm{CFH}$ & $B$ & h042 & 04/04/92 & 900 & 0.67 & - \\
\hline id & id & $R$ & h041 & id & 60 & 0.53 & - \\
\hline id & OHP & $U$ & $2 \mathrm{c} 57$ & $20 / 01 / 91$ & 4200 & 3.25 & - \\
\hline id & id & $B$ & $2 \mathrm{c} 54$ & id & 1000 & 2.90 & 2.18 \\
\hline id & id & $V$ & $2 \mathrm{c} 53$ & id & 120 & 2.06 & 1.95 \\
\hline id & id & $R$ & $2 \mathrm{c} 55$ & id & 120 & 2.49 & 2.07 \\
\hline id & id & $I$ & $2 \mathrm{c} 56$ & id & 150 & 2.28 & 2.12 \\
\hline id & id & $U$ & $6 \mathrm{~b} 20$ & $15 / 01 / 94$ & 3600 & 3.68 & 2.94 \\
\hline
\end{tabular}


Table 3. Second list of frames used in this work. See previous Table for explanations

\begin{tabular}{|c|c|c|c|c|c|c|c|}
\hline NGC & OBS & $\bar{F}$ & File & Date & Exp. & $\overline{W_{0}}$ & $\overline{W_{\mathrm{i}}}$ \\
\hline 4026 & TBL & $B$ & t116 & $07 / 03 / 94$ & 1453 & 1.15 & 0.75 \\
\hline id & id & $V$ & t113 & id & 332 & 1.06 & 0.85 \\
\hline id & id & $R$ & $\mathrm{t} 114$ & id & 360 & 0.91 & 0.76 \\
\hline id & id & $I$ & $\mathrm{t} 115$ & id & 360 & 1.27 & 1.10 \\
\hline id & $\mathrm{OHP}$ & $B$ & $6 \mathrm{~b} 56$ & $17 / 01 / 94$ & 480 & 3.36 & 2.21 \\
\hline id & id & $R$ & $6 \mathrm{~b} 57$ & id & 60 & 2.64 & 2.20 \\
\hline id & id & $I$ & $6 \mathrm{~b} 58$ & id & 60 & 2.87 & 2.15 \\
\hline id & id & V & $6 c 29$ & $18 / 01 / 94$ & 120 & 2.20 & 1.99 \\
\hline id & id & $U$ & $6 c 30$ & id & 3600 & 2.86 & 2.11 \\
\hline 4036 & TBL & B & $\mathrm{t} 120$ & $07 / 03 / 94$ & 1500 & 1.35 & 0.95 \\
\hline id & id & $V$ & t117 & id & 360 & 0.99 & - \\
\hline id & id & $R$ & t118 & id & 360 & 1.05 & - \\
\hline id & id & $I$ & t119 & id & 360 & 1.35 & - \\
\hline id & $\mathrm{OHP}$ & $U$ & $6 c 32$ & $18 / 01 / 94$ & 3000 & 2.86 & 2.35 \\
\hline id & id & $B$ & $1 \mathrm{c} 14$ & $13 / 12 / 90$ & 780 & 2.58 & 2.03 \\
\hline id & id & V & 2a59 & $18 / 01 / 91$ & 180 & 2.35 & 2.04 \\
\hline id & id & V & $6 c 31$ & $18 / 01 / 94$ & 120 & 2.08 & - \\
\hline id & id & $R$ & $1 \mathrm{c} 12$ & $13 / 12 / 90$ & 180 & 2.66 & 1.93 \\
\hline id & id & $I$ & $1 \mathrm{c} 13$ & id & 180 & 2.39 & 2.18 \\
\hline 4111 & TBL & B & t311 & $10 / 03 / 94$ & 1500 & 1.22 & 0.94 \\
\hline id & id & V & t308 & id & 342 & 1.18 & 0.94 \\
\hline id & id & $R$ & t309 & id & 410 & 1.13 & 0.96 \\
\hline id & $\mathrm{OHP}$ & $U$ & $6 c 34$ & $18 / 01 / 94$ & 3600 & 2.81 & 2.01 \\
\hline id & id & B & $2 b 53$ & $18 / 01 / 91$ & 1080 & 2.50 & 1.91 \\
\hline id & id & B & $2 b 54$ & id & 360 & 2.54 & 1.92 \\
\hline id & id & $V$ & $2 \mathrm{~b} 52$ & id & 180 & 2.07 & 1.95 \\
\hline id & id & V & $6 c 33$ & $18 / 01 / 94$ & 180 & 2.07 & 1.95 \\
\hline id & id & $R$ & $2 \mathrm{~b} 56$ & id & 180 & 2.60 & 1.92 \\
\hline id & id & $I$ & $2 \mathrm{~b} 57$ & id & 180 & 2.42 & 1.99 \\
\hline 4350 & TBL & $B$ & t316 & $10 / 03 / 94$ & 1500 & 0.98 & - \\
\hline id & id & V & t313 & id & 360 & 1.29 & - \\
\hline id & id & $R$ & $\mathrm{t} 314$ & id & 360 & 1.30 & - \\
\hline id & id & $I$ & t315 & id & 360 & 1.96 & - \\
\hline id & $\mathrm{OHP}$ & $U$ & $3 \mathrm{a} 3 \mathrm{~s}$ & $15 / 3 / 91$ & 4800 & 2.85 & 2.30 \\
\hline id & id & B & $2 \mathrm{~b} 65$ & $19 / 1 / 91$ & 1200 & 2.63 & 1.88 \\
\hline id & id & $V$ & $2 \mathrm{~b} 64$ & id & 180 & 2.34 & 1.87 \\
\hline id & id & V & $3 a 32$ & $15 / 3 / 91$ & 180 & 2.27 & - \\
\hline id & id & $R$ & $2 \mathrm{~b} 66$ & $19 / 1 / 91$ & 180 & 2.54 & 1.87 \\
\hline
\end{tabular}

Table 4. Third list of frames used in this work. See previous Table for explanations

\begin{tabular}{llllllll}
\hline NGC & OBS & $F$ & File & Date & Exp. & $W_{0}$ & $W_{\mathrm{i}}$ \\
\hline 5308 & TBL & $B$ & $\mathrm{t} 124$ & $07 / 03 / 94$ & 1500 & 1.07 & - \\
id & id & $V$ & $\mathrm{t} 121$ & id & 360 & 0.94 & - \\
id & id & $R$ & $\mathrm{t} 122$ & id & 360 & 1.00 & - \\
id & id & $I$ & $\mathrm{t} 123$ & id & 360 & 1.45 & - \\
id & OHP & $B$ & $3 \mathrm{a} 39$ & $15 / 03 / 91$ & 900 & 1.96 & 1.84 \\
id & id & $V$ & $3 \mathrm{a} 37$ & id & 120 & 2.06 & - \\
id & id & $V$ & $3 \mathrm{a} 40$ & id & 300 & 2.05 & - \\
id & id & $R$ & $3 \mathrm{a} 41$ & id & 300 & 2.10 & 1.87 \\
id & id & $I$ & $3 \mathrm{a} 42$ & id & 300 & 1.68 & - \\
5422 & TBL & $V$ & $\mathrm{t} 125$ & $07 / 03 / 94$ & 360 & 0.85 & - \\
id & id & $R$ & $\mathrm{t} 126$ & id & 360 & 0.81 & - \\
id & id & $I$ & $\mathrm{t} 127$ & id & 360 & 1.32 & - \\
id & OHP & $B$ & $3 \mathrm{c5} 5$ & $18 / 03 / 91$ & 720 & 3.11 & 2.08 \\
id & id & $V$ & $3 \mathrm{c} 53$ & id & 300 & 3.02 & 1.94 \\
id & id & $R$ & $3 \mathrm{c} 54$ & id & 300 & 2.89 & 1.95 \\
id & id & $I$ & $3 \mathrm{c} 55$ & id & 300 & 2.62 & 2.01 \\
7332 & TBL & $B$ & $\mathrm{td} 74$ & $16 / 08 / 93$ & 1200 & 1.65 & - \\
id & id & $R$ & td72 & id & 600 & 2.41 & 1.60 \\
id & OHP & $B$ & $1 \mathrm{c} 31$ & $13 / 12 / 90$ & 720 & 3.02 & 2.15 \\
id & id & $V$ & $1 \mathrm{c} 30$ & id & 240 & 3.03 & 2.16 \\
7457 & CFH & $B$ & h04 & $22 / 10 / 90$ & 240 & 0.77 & - \\
id & id & $R$ & h02 & id & 60 & 0.56 & - \\
id & OHP & $B$ & $1 \mathrm{a} 3$ & $10 / 12 / 90$ & 900 & 3.23 & - \\
id & id & $V$ & $1 \mathrm{a} 4$ & id & 450 & 3.01 & - \\
\hline
\end{tabular}

1011 pixels. Note however that the data for NGC 3115 were obtained in 1989 with the Cassegrain camera at the same scale and field. With the HRCam, a guiding star has to be located at a specific place in the field, so that the studied object is often far from centred, and the usable galaxian range may be rather limited: the range of measurable majA values is $30-$ 50 arcsec. Unfortunately, only the $B$ and $R$ colours are available, with $R$ according to Cousins. Stellar images give a mean $F W H M$ of $0.66^{\prime \prime}$ in $B$ and $0.57^{\prime \prime}$ in $R$.

2. Obervations of fair resolution were obtained by one of us for 6 more objects, at the $2 \mathrm{~m}$ Bernard Lyot telescope of the Pic du Midi (TBL). With the catadioptric focal reducer, the scale was $0.242 \mathrm{arcsec} / \mathrm{pixel}$, and the usable field $951 \times 1001$ pixels. Frames in $B, V, R$ and $I$ were generally obtained. Some problems with the optical adjustements of the camera occured in 1994, so that the PSF is seen to vary significantly in the field, while the PSF's in $I$ are rather poor, as compared to the other bands. Several frames from this run are affected by geometrical errors (of electronic origin), involving some limitations in their possible uses. The mean $F W H M$ 's from stellar images are $1.15^{\prime \prime}$ in $B$, $1.05^{\prime \prime}$ in $V, 1.03^{\prime \prime}$ in $R$ and $1.47^{\prime \prime}$ in $I$ for the main run of 1994, while the observations of NGC 7332 are less good.

3. The above material was completed by frames from the series obtained at the $120 \mathrm{~cm}$ of the Observatoire de Haute-Provence (OHP) by J. Marchal and one of us. In 1990-91, the available scale was $0.84 \mathrm{arcsec} / \mathrm{pixel}$, and the usable field $321 \times 511$ pixels, or about $4 \times$ 7 arcmin. Later a camera with $0.76 \mathrm{arcsec} / \mathrm{pixel}$ and a field of about $7 \times 7$ arcmin became available, and was used in our 1994 observing run. Frames in $U, B, V, R$ and $I$ were obtained, but part of the $U$ images were discarded due to unsufficient $\mathrm{S} / \mathrm{N}$. The seeing at the OHP is generally in the $2-3$ arcsec but may become still worse!

\section{Data analysis}

\subsection{General indications}

The here used techniques of data reduction and colour measurements have been extensively described in Paper IV of this series (Michard 1999). The indications given there about the preliminary steps, the $1 \mathrm{D}$ and $2 \mathrm{D}$ colour reductions, the calibrations and usual corrections of the colours remain largely unchanged. Here is given a summary of a few important points:

1. The isophotal analysis according to Carter (1978) is a necessary step of our procedure. A set of isophotes, in parametric form, is used to locate the points to be measured to derive local magnitudes and colours, 
and define adequate averaging ranges to obtain a sufficient $\mathrm{S} / \mathrm{N}$ without unwanted degradation of the resolution. The measured contours are then 0.1 mag distant, and they are symmetrized by simplifications in their Carter's representation: an invariant center is adopted, and odd harmonics are put to zero.

2. Radial 1D SuBr or colour distributions are obtained separately for each axis, and also for each opposite half of a given axis. To define data points for a given axis, pixels are averaged within a range of \pm 22.5 around the nominal direction, this range being defined by the eccentric anomaly $\omega$ of the Carter Reference Ellipse. Note that a given range in $\omega$ corresponds to different ranges of polar angle at the majA and minA, depending upon the $q$ ratio of the isophote. For instance, for a flat galaxy with $q=.3$ the adopted range of \pm 22.5 in $\omega$ will correspond to $\pm 7^{\circ} .1$ in polar angle at the majA and to $\pm 54^{\circ}$ at the minA. Examples of such detailed radial profiles are shown below in Fig. 3 .

Besides this we have obtained average radial colour profiles, giving the mean isophotal colour against the mean isophotal radius (see plots in the Appendix).

3. Azimuthal colour distributions have been systematically measured. These are obtained using all pixels within "measurements rings" limited by symmetrized isophotal contours, again defined by the Carter's representation and labelled by their inner and outer radii $r$. In azimuth there are 121 data points taken at equal steps in the eccentric anomaly $\omega$ along Carter's Reference Ellipse. These are usually plotted at the relevant polar angle, 0 and $180^{\circ}$ for the majA, 90 and $270^{\circ}$ for the minA. For strongly elongated objects, the points are thus much more closely packed near the major than the minor axis as may be noted in published diagrams (Figs. 1, 2).

4. 2D colour maps have been produced from the best pairs of frames, using the usual definition of the colour as a difference of magnitudes. These have been used to produce the 12 maps assembled in Figs. A1 to A12, and the FITS images made available upon request to interested readers.

5. Again special care has been taken of correcting, at least approximately, the important errors in colours resulting from "differential seeing", that is the different PSF's of the two frames involved in measuring a colour. Some new features have been introduced in this part of the present work and will be now described.

\subsection{PSF matching with improved resolution}

As pointed out in Paper IV, the first step in attempting to equalize the PSF's of two different frames, is to determine a correcting function FC, such that the convolution of the sharper of the two PSF's with FC will reproduce the other one. Then one can convolve the "sharp" frame by
FC to match its new PSF to the one of the "unsharp" one. Accordingly the colour is observed with the resolution of the worse frame.

In principle, one could also deconvolve with $\mathrm{FC}$ the worse of the two frames, to match its PSF with the best one of the pair. In this case one has to be aware of the artefacts introduced by "ringing" of the solution, as experimented by Michard (1996) for the widely used Lucy's deconvolving routine. If $\mathrm{FC}$ is, however, much narrower than the original PSF such artefacts may remain negligible. A test is available, as the deconvolution by FC may be applied to a stellar image: in this case ringing will manifest itself by a darker ring around the deconvolved image.

It has been attempted to generalize the above considerations by determining the FC functions needed to bring the PSF's of a group of images of similar resolutions to a single narrower common PSF, indeed a Gaussian of suitable $F W H M$. Sometimes the best PSF in the group was used for matching instead of a Gaussian. The results of this exercize are given in Tables 1, 2, 3 under the label of "improved $F W H M$ ". It may be noted that we suceeded in matching the PSF's to very similar width's in most cases, and with noticeable improvements of between 20 to $33 \%$ of their width.

The eventual effect of deconvolution artefacts was checked by performing the isophotal analysis upon both the original and "improved" frames: the parameters $q$ and $e_{4}$ provide very sensitive tests. Other tests are feasible by comparing the colours measured from pairs of frames matched by either convolution or deconvolution: if new colour features occur with the "improved" frames, one should be cautious!

\subsection{Errors in the study of near edge-on disk objects}

The errors induced by seeing in colour studies of Etype objects have been discussed at some length in our Paper IV, and in the previous papers by several authors quoted there. The errors may be very large in the central part of such objects, involving a strong light gradient, but at radial distances larger than a few times the seeing disk, the measurements are hopefully unbiased. This will not be the case however for objects embodying a thin disk at nearly edge-on projection. The "transverse" light gradient in such objects, that is parallel to the minA, is then large, and the colour badly affected through most of the minA length. We have made a number of numerical experiments relevant to this question, specially in connection with the use of our OHP data to supplement the frames of much better resolution obtained at the CFHT and the TBL. The "OHP seeing" leads to reduced transverse visiblity of the disk in edge-on objects, and reduced contrast of the disk colour (if different from the bulge one). Since part of our OHP frames have elongated and asymetric PSF's in the $\alpha$ direction, due to poor performance of the autoguider, the effects may be dramatic for edge-on objects 
of unfavourable PA! A list of the main causes of errors encountered in this work is offered here, besides the trivial ones of photometric noise (negligible except far from galaxian centers), and calibrations errors (of little importance for our purposes):

- Improper PSF matching leaves important systematic errors in observed colours both near the galaxian center and along the minA of near edge-on disk galaxies;

- PSF asymmetries may also give important errors in minA light and colour profiles, unless they are very similar in both the frames used. Such asymmetries, specially in the outer wings of the PSF, are not readily corrected by our techniques of PSF matching;

- The background will lead to large errors in colours at low light levels: these will come in part from uncertainties in the adopted sky background constants, but also from residual background fluctuations and trends. These are no doubt present after the flat-fielding and other correction techniques, and they were large with one of the used OHP CCD's, at least in the $I$ band. A poor choice of the average background level also results in calibration errors;

- The unsufficient resolution of the frames, even if properly matched, leads to important errors if sharp colour features are present: this is certainly the case near the galaxian center, and for many dust patterns.

\subsection{Evaluation of various errors from multiple observations}

If two or more frames of a given object in a given band $C$ are available, one may evaluate the pseudo-colour $C-C$ from such pairs. These are controlled by the measurement errors of various origins listed above. The 4th one, i.e. errors due to unsufficient resolution, do not enter however if the two frames are from the same observatory and therefore similar resolution (after PSF matching).

Errors in CFHT observations have been discussed in our previous Paper IV, from 8 pairs of duplicate observations of mostly elliptical objects, plus NGC 3115. This study has been repeated with emphasis upon objects containing a thin disk, such as NGC 3115, 3377, 3610. The analysis of duplicate frames was conducted in such a way as to derive errors upon the quantities to be considered in the present study.

A number of duplicate observations also occur in our collection of OHP frames. Because our sample mostly contains very flat galaxies, with very large transverse light gradients, the seeing effects are quite large. The experiments with duplicate OHP frames led to the rejection of part of the results from this material, except where it could supplement the higher resolution data without introducing unwanted bias.

Another technique is needed to estimate errors in the TBL data. It should be noted that there are 6 galaxies in common between the TBL and OHP series: the comparison of measurements from these two sources gives indications upon errors in both, and, together with the above noted experiments upon duplicate frames in the OHP material, opens a way to estimate a set of errors for both sources. It should be emphasized however that errors are mostly systematic, leaving no hope to estimate these by straightforward statistics. The two applied techniques, that is pseudo-colours $C-C$ from duplicate frames on the one hand, and comparisons of colours from different sources on the other, are not equally sensitive to the various systematic errors listed above. For instance, near centre colour features are much sharper at 1 arcsec resolution than with the OHP seeing: this introduces large local differences between the colour distributions of the TBL and OHP series.

Probable errors derived by the above techniques are summarized in Tables 5 and 6, separately for the three sources of material and various quantities of interest. In Tables 5 are given the errors for radial colour profiles. First appear the errors for the separate semi- majA an minA as in Fig. 3 (noted $\sigma_{13}, \sigma_{24}$ ). For OHP material $\sigma_{24}$ is much larger than $\sigma_{13}$ as expected from poor PSF matching. Then come the errors $\sigma_{\mathrm{l}}, \sigma_{\mathrm{s}}$ for mean colours along the majA and minA respectively: they are smaller mainly because the effects of PSF asymmetries cancel out. Finally is given the estimated mean errors $\sigma_{\mathrm{r}}$ for the isophotal radial colour profiles.

Table 6 contains estimated mean errors for azimuthal colour profiles. Again errors $\sigma_{\mathrm{a}}$ for the tips of the majA and $\sigma_{\mathrm{c}}$ for the arcs in the $\min \mathrm{A}$ regions are distinguished: such errors are expected to apply to such graphs as in Figs. 1, 2. We also give the error $\sigma_{\mathrm{D}}$ expected for the colour differences between majA and minA such as tabulated in Table 10.

The above estimates were mainly obtained for $B-V$ and $B-R$ colours, while errors are probably larger for $U-V$ and $V-I$. The contrast of E-S0 galaxies against sky background is reduced by roughly $1 \mathrm{mag}$ in $U$ and $0.5 \mathrm{mag}$ in $I$ (greatly varying with zenith distance!). Besides this, the $\mathrm{S} / \mathrm{N}$ ratio is poor for part of our $U$ OHP frames, and the background unclean for part of the $I$ frames of the same source. Larger mean OHP $\sigma_{\mathrm{r}}$ errors for $U-V$ and $V-I$ were "estimated" and are entered in the relevant plots.

Finally the standard error upon the colour gradients in Tables 7 and 8 has been evaluated to 0.03 from a comparison between the various data series.

\subsection{Calibrations and calibration errors}

The frames were calibrated from the $U B V$ aperture photometry results compiled in the catalogues by Longo \& de Vaucouleurs $(1983,1985)$, and the $R I$ results in de Vaucouleurs and Longo (1988). For NGC 3115 and 4350 the more recent data by Poulain (1988) was prefered. Since 
Table 5. Estimates of mean errors in radial colour profiles from duplicate frames. The columns refer to ranges of measurement, i.e. $C_{0}$, the central region inside the approximate $F W H M, 2 W$, a small range near a radius $r$ about twice the $F W H M$, and the $\mu_{V}$ of $V$ magnitude ranges, roughly 1 mag broad. The first two values are intended to describe the errors associated with resolution problems (after PSF matching), the other to errors associated with background problems (choice of mean level and residual fluctuations). The succesive lines are $\sigma_{13}, \sigma_{24}$ the mean errors for a data point in one of the semi-majA and -minA respectively as in Fig. $3 ; \sigma_{1}, \sigma_{\mathrm{s}}$, the mean errors for the average colours along the majA or minA respectively; and finally $\sigma_{\mathrm{r}}$ for the data points of the radial colour profiles of Figs. A1 to A12. A colon stands for values of lower weight

\begin{tabular}{lllllllllll}
\hline Obs. & $C_{0}$ & $2 W$ & 17 & 18 & 19 & 20 & 21 & 22 & 23 & 24 \\
\hline CFH $\sigma_{13}$ & - & .012 & .010 & .016 & .042 & $.055:$ & - & - & - & - \\
CFH $\sigma_{24}$ & - & .023 & .018 & .015 & .030 & $.043:$ & - & - & - & - \\
CFH $\sigma_{1}$ & - & .009 & .008 & .011 & .032 & $.040:$ & - & - & - & - \\
CFH $\sigma_{\mathrm{s}}$ & - & .022 & .018 & .013 & .028 & $.030:$ & - & - & - & - \\
CFH $\sigma_{\mathrm{r}}$ & .026 & .015 & .012 & .012 & .028 & $.036:$ & - & - & - & - \\
\hline TBL $\sigma_{13}$ & - & - & - & .020 & .015 & .015 & .015 & .035 & .070 & - \\
TBL $\sigma_{24}$ & - & - & - & .035 & .030 & .030 & .030 & .040 & .070 & - \\
TBL $\sigma_{1}$ & - & - & - & .015 & .010 & .010 & .015 & .025 & .055 & - \\
TBL $\sigma_{\mathrm{s}}$ & - & - & - & .035 & .030 & .020 & .020 & .035 & .055 & - \\
TBL $\sigma_{\mathrm{r}}$ & .035 & - & - & .020 & .015 & .010 & .015 & .030 & .055 & - \\
\hline OHP $\sigma_{13}$ & - & .022 & - & - & .020 & .020 & .025 & .030 & .055 & .090 \\
OHP $\sigma_{24}$ & - & .043 & - & - & .050 & .040 & .045 & .060 & .060 & .085 \\
OHP $\sigma_{1}$ & - & .013 & - & - & .010 & .015 & .015 & .020 & .040 & .075 \\
OHP $\sigma_{\mathrm{s}}$ & - & .035 & - & - & .035 & .025 & .030 & .035 & .045 & .070 \\
OHP $\sigma_{\mathrm{r}}$ & .050 & .019 & - & - & .020 & .010 & .010 & .020 & .030 & .055 \\
\hline
\end{tabular}

Table 6. Estimates of mean errors in azimuthal colour profiles from duplicate frames. The columns refer to the successive "measurements rings" introduced in 3.1.3. In succesive lines are given the mean errors $\sigma_{\mathrm{a}}$ and $\sigma_{\mathrm{c}}$ for data points in near majA and near $\min A$ regions respectively, in graphs like Figs. 11, 12, or 14. The $\sigma_{\mathrm{D}}$ are mean errors for such data as Table 9, i.e. colour differences between majA and minA at a given isophote

\begin{tabular}{llllllll}
\hline Obs. & $2 .-3.2$ & $3.2-5$. & $5 .-7.9$ & $7.9-12.6$ & $12.6-20$. & $20 .-31.6$ & $31.6-50$. \\
\hline CFH $\sigma_{\mathrm{a}}$ & .018 & .017 & .021 & .043 & .062 & - & - \\
CFH $\sigma_{\mathrm{c}}$ & .025 & .018 & .023 & .036 & .061 & - & - \\
CFH $\sigma_{\mathrm{D}}$ & .018 & .011 & .012 & .016 & .026 & - & - \\
\hline TBL $\sigma_{\mathrm{a}}$ & - & .02 & .02 & .03 & .045 & .06 & - \\
TBL $\sigma_{\mathrm{c}}$ & - & .03 & .03 & .04 & .055 & .07 & - \\
TBL $\sigma_{\mathrm{D}}$ & - & .02 & .02 & .02 & .03 & .04 & - \\
\hline OHP $\sigma_{\mathrm{a}}-$ & - & - & .017 & .026 & .041 & .103 \\
OHP $\sigma_{\mathrm{c}}-$ & - & - & .056 & .076 & .105 & .087 \\
OHP $\sigma_{\mathrm{D}}$ & - & - & - & .033 & .033 & .036 & .051 \\
\hline
\end{tabular}

aperture photometry data in $R$ and $I$ are missing for many of the studied objects, they were replaced by (very tight) correlations between $V-R$ and $V-I$ against $B-V$, derived from Poulain's photometry of early-type galaxies.

Errors in calibrations should be added to the errors analysed above. They are of course closely bound to the availability and accuracy of calibration data. These are abundant for NGC 2549, 3115, 4111, 4350, 7332, 7457, but rather scanty for NGC 3098, 4036, 5308, 5422. The data are best in $B-V$, and less good in $U-V$ (due to scarcer data and poorer $\mathrm{S} / \mathrm{N}$ in $U$ ), or $V-I$ (due to the use of an ancillary correlation with $B-V$ ). It is believed that calibration errors reduce to 0.01 in the best cases and may reach 0.04 in the worse.

Remark: The survey in BP94 includes minA colour profiles for 4 objects of the present sample. These authors obtained their own photometry by observing stan- dard stars (although in rather unsatisfactory conditions). To quote their paper, their "photometric accuracy is estimated to be better than $0.05 \mathrm{mag}$ in $R$ and $I$ and $0.10 \mathrm{mag}$ in $B$ and $U$ ". We could compare results for 7 $B-R$ or $R-I$ colours measured at the same place, i.e. $s=5$ arcsec along the minA. We find for Them-Us a mean of .02 and a $\sigma$ of 0.05 , well in line with estimated calibrations errors in BP94 and above.

Incidentally we also compared minA gradients (measured in identical ranges). These are in good agreement for NGC 5422 and 7457, but not for 5308 and 7332: in these two objects BP94 gradients (their Table 2) are anomalous, $B-R$ increasing outwards instead of decreasing as usual. This might be an effect of "differential seeing" if the $R$ frame was distinctly sharper than the $B$ one. 


\subsection{Corrections to observed colours}

The total colour corrections $C C$ for galactic extinction and $\mathrm{K}$-effect, are based upon the galactic extinction coefficient $A_{\mathrm{g}}$ and the radial velocities $V_{\text {opt }}$ given in the RC3 (de Vaucouleurs et al. 1991). The following corrections have been adopted, in the 4 used colours:

$$
\begin{aligned}
& \text {. in } B-V, C C=A_{\mathrm{g}}(1 / 4.3)+0.09910^{-4} V_{\mathrm{opt}} \\
& \text {. in } V-I, C C=A_{\mathrm{g}}(1.40 / 4.3)+0.04510^{-4} V_{\mathrm{opt}} \\
& \text {. in } B-R, C C=A_{\mathrm{g}}(1.75 / 4.3)+0.12610^{-4} V_{\mathrm{opt}} \\
& \text {. in } U-V, C C=A_{\mathrm{g}}(1.70 / 4.3)+0.08410^{-4} V_{\mathrm{opt}} \text {. }
\end{aligned}
$$

The coefficients for galactic reddening take into account the approximate passbands used in our observations, that is Johnson's $U, B$ and $V$, Cousins's $R$ and $I$. The corrections have been applied only to tabulated results, not to graphical data dealing with individual galaxies.

\section{Observational results}

\subsection{General description}

The examination of the data in various $1 \mathrm{D}$ or $2 \mathrm{D}$ displays, showed that the colour distributions in the studied S0's are determined by several effects:

1. To a first (rough) approximation the colours are constant upon a given isphote and decrease monotonuously with increasing isophotal radius, as occurs in Etype galaxies;

2. Obvious dust patterns occur in a number of objects, giving rise to high contrast colour features;

3. Diffuse dust may be concentrated in disks, leading to reddening of the disk as compared to the adjoining spheroid, or sometimes to the minor axis asymmetry in light and colour, previously discussed by Michard \& Simien (1993) (MS93);

4. Since, from population effects alone, bulge and disk may have somewhat different mean colours, and possibly have different radial colour gradients, deviations of the isochromes from the isophotes will occur. It turns out that the disk dominated major axis often becomes redder, far from center, than the minor axis for a given isophote: this may be tentatively ascribed to a larger colour gradient in the bulge, this component blueing outwards more than does the disk. On the other hand a stretch of bluer major axis (bluer disk!) appears in two of the studied objects.

\subsection{Radial colour profiles}

\subsubsection{Mean isophotal colours}

In the Appendix (Figs. A1 to A12), the distribution of mean colours for consecutive isophotes are shown against the isophotal radius $r$ in a logarithmic scale. The 4 colours
$B-V, V-I, B-R$ and $U-V$ are available for 8 objects; $U-V$ is missing in the data for NGC 5308, 5422, 7332 and 7457, and $V-I$ also for the two latter. Both the OHP low resolution data and the high resolution data from CFHT and TBL are shown. The examination of the graphs suggests the following comments:

1. The agreement between colour profiles from the different instruments is generally fair. The deviations resulting from different resolutions are lessened by introducing adequate inner cutoffs for various observations, but may remain large in cases where a minute red feature occurs near the galaxian center as in NGC 4111. Disagreements at large $r$ are obviously due to errors in the sky background corrections. Some differences between measurements from wide field and low field exposures may come from the calibrations, because the set of aperture photometry data will not necessarily be the same in such a case: an example of this situation is the $B-R$ graph for NGC 3115 ;

2. The radial colour profiles are rather similar for the various objects, but there are important systematic changes of the gradients in the various colours. $V-I$ is always the flatter profile, then $B-V, B-R, U-V$ have stronger and stronger gradients. The comparison of colour gradients for different colours is of interest in view of the uncertainties in the origin of this property;

3. The inner colour profiles, as measured with our higher resolution vary widely from object to object: for instance, NGC 2549 shows a rather flat colour run, while NGC 3245, 4026 or 4111 have a central red peak. This is essentially due to the presence of various dust concentrations in the central galaxian regions. Such features may be largely washed out by lack of resolution. In Table 7 are given the mean colours at $r_{\mathrm{e}}$ and the logarithmic gradients $\Delta C / \Delta \log r$, where $C$ is any of the colours under study. As expected these parameters show significant variations from galaxy to galaxy. It is perhaps suggestive to consider the "statistics" of gradients for each colour:

in $B-V, 12$ cases, mean $=0.072, \sigma=.041$
in $V-I, 10$ cases, mean $=0.045, \sigma=.029$
in $B-R, 11$ cases, mean $=0.104, \sigma=.047$
in $U-V, 8$ cases, mean $=0.224, \sigma=.031$.

\subsubsection{Bulge colours and gradients}

There are two ways to distinguish the colours of the different components in S0's, say the bulge and the disk: on the one hand, it is possible to analyse the images in terms of these two components, independently for the two pass bands; on the other hand one can look at such parts of the images where one of the component strongly dominates the other. The first type of analysis will give model dependent results; in the second technique some "pollution" of the bulge light by the disk may be feared (or conversely). Since the bulge stands out very clearly along the 
Table 7. Reference colours and gradients, separately for mean isophotes and bulge dominated regions respectively. Very uncertain values are labelled with a colon. The sign of gradients $\Delta C / \log r$ has been changed. Obs: source of observations. $r_{\mathrm{m}}$ mean isophotal radius in arcsec of colour measurement. $r_{1}, r_{2}$ : range for estimating gradients. Com: "component", as Iso for mean isophotal colours, Bul for bulge. In the first case the measurement radius is $r_{\mathrm{e}} . B-V$, etc.: mean local colours. $\Delta$ : colour gradients. Notes: Scarce calibration data for NGC 3098, 5308, 5422. NGC 2549: bulge not measured, as not prominent enough against disk. NGC 3098: small bulge not measurable from OHP frames. NGC 3245: bulge not measured, as not prominent enough against disk. NGC 4026: bulge measured on W side, as E side is dusty. NGC 4036: bulge measured on N side, as $\mathrm{S}$ side is very dusty; isophotal gradients probably affected by the strong inner dust features. NGC 4111: bulge not measured, as affected by dust pattern. NGC 5308: bulge not measurable from OHP frames. NGC 5422: bulge measured on W side, as E side is dusty; isophotal gradients probably affected by the strong inner dust features. NGC 7332: large bulge measured on majA to avoid dust pattern. NGC 7457: $B-R$ data taken at $r_{\mathrm{e}} / 4$ from low fields CFHT frames; bulge not seen

\begin{tabular}{|c|c|c|c|c|c|c|c|c|c|c|c|c|}
\hline NGC & Obs. & Com. & $r_{\mathrm{m}}$ & $r_{1}, r_{2}$ & $B-V$ & $\Delta$ & $V-I$ & $\Delta$ & $B-R$ & $\Delta$ & $U-V$ & $\Delta$ \\
\hline 2549 & - & Iso & 11 & 3,30 & $0.92 \pm .02$ & .06 & $1.20 \pm .02$ & .04 & $1.48 \pm .02$ & .09 & $1.45 \pm .02$ & .24 \\
\hline 3098 & - & Iso & 8 & 3,20 & $0.80 \pm .03$ & .10: & $1.16 \pm .03$ & .01 & $1.38 \pm .03$ & .07 & $1.36 \pm .03$ & .20 \\
\hline 3098 & $\mathrm{CFH}$ & Bul & 3 & 1,5 & - & - & - & - & $1.42 \pm .02$ & .06 & - & - \\
\hline 3115 & - & Iso & 30 & 3,40 & $0.95 \pm .01$ & .05 & $1.21 \pm .01$ & .00 & $1.52 \pm .01$ & .08 & $1.43 \pm .02$ & .17 \\
\hline 3115 & $\mathrm{CFH}$ & Bul & 5 & 3,8 & - & - & - & - & $1.52 \pm .02$ & .08 & - & - \\
\hline 3115 & $\mathrm{OHP}$ & Bul & 10 & 8,20 & $0.96 \pm .03$ & .07 & $1.23 \pm .03$ & - & $1.52 \pm .03$ & .08 & $1.49 \pm .03$ & .18 \\
\hline 3245 & - & Iso & 21 & 3,30 & $0.87 \pm .01$ & .05 & $1.14 \pm .01$ & .05 & $1.39 \pm .01$ & .07 & $1.38 \pm .02$ & .22 \\
\hline 4026 & - & Iso & 16 & 3,30 & $0.90 \pm .01$ & .04 & $1.18 \pm .01$ & .05 & $1.47 \pm .01$ & .06 & $1.42 \pm .01$ & .21 \\
\hline 4026 & TBL & Bul & 10 & 4,15 & $0.88 \pm .02$ & .06 & $1.18 \pm .02$ & .05 & $1.44 \pm .02$ & .09 & - & - \\
\hline 4026 & $\mathrm{OHP}$ & Bul & 10 & 4,15 & $0.89 \pm .02$ & .06 & $1.20 \pm .02$ & .04 & $1.46 \pm .02$ & .08 & $1.40 \pm .02$ & .26 \\
\hline 4036 & - & Iso & 20 & 3,30 & $0.90 \pm .01$ & .12 & $1.16 \pm .01$ & $.07:$ & $1.45 \pm .01$ & .15 & $1.46 \pm .01$ & .26 \\
\hline 4036 & TBL & Bul & 10 & 5,20 & $0.89 \pm .02$ & .11 & $1.11 \pm .02$ & .05 & $1.43 \pm .02$ & .11 & - & - \\
\hline 4036 & OHP & Bul & 10 & 5,20 & $0.90 \pm .02$ & .07 & $1.17 \pm .02$ & .04 & $1.46 \pm .02$ & .09 & $1.47 \pm .02$ & .22 \\
\hline 4111 & - & Iso & 10 & 4,25 & $0.83 \pm .02$ & .08 & $1.12 \pm .02$ & .03 & $1.40 \pm .02$ & .10 & $1.23 \pm .03$ & .23 \\
\hline 4350 & - & Iso & 15 & 3,30 & $0.91 \pm .01$ & .10 & $1.21 \pm .01$ & .08 & $1.48 \pm .01$ & .13 & $1.40 \pm .03$ & .26 \\
\hline 4350 & TBL & Bul & 10 & 3,15 & $0.85 \pm .02$ & .12 & - & - & $1.49 \pm .02$ & .18 & - & - \\
\hline 4350 & $\mathrm{OHP}$ & Bul & 10 & 5,15 & $0.92 \pm .02$ & .13 & $1.14 \pm .02$ & .05 & $1.47 \pm .03$ & .17 & $1.43 \pm .03$ & .33 \\
\hline 5308 & - & Iso & 13 & 3,30 & $0.88 \pm .01$ & .08 & $1.17 \pm .01$ & .03 & $1.43 \pm .01$ & .08 & - & - \\
\hline 5308 & TBL & Bul & 3 & 2,5 & $0.90 \pm .03$ & .17 & - & - & $1.43 \pm .03$ & .17 & - & - \\
\hline 5422 & - & Iso & 13 & 3,30 & $0.94 \pm .01$ & .15 & $1.20 \pm .01$ & .09 & $1.54 \pm .01$ & .21 & - & - \\
\hline 5422 & TBL & Bul & 5 & 3,10 & - & - & $1.15 \pm .03$ & .10 & - & - & - & - \\
\hline 5422 & OHP & Bul & 8 & 5,12 & $0.93 \pm .04$ & .14 & $1.19 \pm .04$ & .08 & $1.50 \pm .04$ & .14 & - & - \\
\hline 7332 & - & Iso & 14 & 3,30 & $0.85 \pm .02$ & .02 & - & - & $1.37 \pm .02$ & .09 & - & - \\
\hline 7332 & TBL & Bul & 8 & 3,12 & - & - & - & - & $1.38 \pm .03$ & $.09:$ & - & - \\
\hline 7457 & - & Iso & 33 & 3,30 & $0.84 \pm .02$ & .01 & - & - & $1.39 \pm .02$ & .07 & - & - \\
\hline
\end{tabular}

minA of the near edge-on galaxies of the present sample, we have measured the corresponding colours and gradients, in such cases where the bulge appeared sufficienly clean of dust patterns. An analysis in terms of bulge and disk components is available for nearly all objects studied here (Michard 1998 and unpublished) (M 98), and the results were used to check that the disk contribution, along the minA, in the range selected for defining the bulge, was smaller than about 20\%. For NGC 4026, 4036 and 5422, only this side of the bulge apparently not affected by dust was used. For NGC 7332, the minA is affected on both sides by the dust pattern: we tried to measure the bulge in a strech of the majA where the disk remains negligible, judging from the $e_{4}$ Carter coefficient. The bulge is not dominant enough for measurement in NGC 2549, 3245 and 7457; it is badly affected by dust in NGC 4111 .

Since the axis ratio of bulges is certainly not constant, according to such bulge+disk decompositions where this 
Table 8. Selected measurements of disk colours and colour gradients. The sign of gradients $\Delta C / \log l$ has been changed. Obs: source of observations. $l_{\mathrm{m}}$ : Mean majA of bulge colour estimates in arcsec. $l_{1}, l_{2}$ : range for estimating gradients. $B-V$, etc.: mean local colours. $\Delta$ : disk colour gradients against majA $l$. Notes: NGC 3115: Disk not measured, as nowhere dominant enough. NGC 4036: Disk not distinguished from "mean". NGC 7457: Disk not distinguished from "mean"

\begin{tabular}{|c|c|c|c|c|c|c|c|c|c|c|c|c|}
\hline NGC & Obs. & Com. & $l_{\mathrm{m}}$ & $l_{1}, l_{2}$ & $B-V$ & $\Delta$ & $V-I$ & $\Delta$ & $B-R$ & $\Delta$ & $U-V$ & $\Delta$ \\
\hline 2549 & $\mathrm{OHP}$ & Dis & 40 & 20,50 & $0.92 \pm .02$ & .05 & $1.18 \pm .02$ & .00 & $1.47 \pm .02$ & .06 & $1.44 \pm .02$ & .18 \\
\hline 3098 & $\mathrm{CFH}$ & Dis & 20 & 7,25 & - & - & - & - & $1.38 \pm .01$ & .04 & - & - \\
\hline 3098 & $\mathrm{OHP}$ & Dis & 20 & 7,25 & $0.80 \pm .02$ & .02 & $1.16 \pm .02$ & .02 & $1.37 \pm .02$ & .04 & $1.35 \pm .03$ & .06 \\
\hline 3245 & $\mathrm{OHP}$ & Dis & 30 & 10,40 & $0.87 \pm .02$ & .04 & $1.14 \pm .02$ & .01 & $1.40 \pm .02$ & .06 & $1.40 \pm .03$ & .09 \\
\hline 4026 & TBL & Dis & 40 & 25,60 & $0.91 \pm .02$ & .05 & $1.18 \pm .02$ & .00 & $1.47 \pm .02$ & .07 & - & - \\
\hline 4026 & $\mathrm{OHP}$ & Dis & 40 & 25,60 & $0.90 \pm .02$ & .08 & - & - & $1.47 \pm .02$ & .09 & $1.46 \pm .02$ & .12 \\
\hline 4111 & TBL & Dis & 40 & 25,50 & $0.82 \pm .02$ & .04 & - & - & $1.31 \pm .02$ & .07 & - & - \\
\hline 4111 & $\mathrm{OHP}$ & Dis & 40 & 25,50 & $0.80 \pm .02$ & .02 & $1.09 \pm .02$ & -.03 & $1.32 \pm .02$ & .02 & $1.15 \pm .02$ & .11 \\
\hline 4350 & TBL & Dis & 20 & 13,34 & $0.90 \pm .02$ & .04 & - & - & $1.55 \pm .02$ & .09 & - & - \\
\hline 4350 & $\mathrm{OHP}$ & Dis & 20 & 13,34 & $0.91 \pm .02$ & .08 & $1.20 \pm .02$ & .03 & $1.50 \pm .02$ & .04 & $1.53 \pm .02$ & .09 \\
\hline 5308 & TBL & Dis & 25 & 15,40 & $0.92 \pm .02$ & .05 & $1.17 \pm .02$ & .05 & $1.46 \pm .02$ & .05 & - & - \\
\hline 5308 & $\mathrm{OHP}$ & Dis & 25 & 15,40 & $0.89 \pm .02$ & .03 & $1.14 \pm .02$ & .03 & $1.43 \pm .02$ & .03 & $1.44 \pm .03$ & .10 \\
\hline 5422 & TBL & Dis & 30 & 25,40 & - & - & $1.21 \pm .02$ & .12 & - & - & - & - \\
\hline 5422 & OHP & Dis & 30 & 25,40 & $0.92 \pm .02$ & .08 & $1.20 \pm .02$ & .13 & $1.50 \pm .02$ & .07 & - & - \\
\hline 7332 & TBL & Dis & 40 & 20,50 & - & - & - & - & $1.33 \pm .02$ & .07 & - & - \\
\hline 7332 & $\mathrm{OHP}$ & Dis & 40 & 20,50 & $0.85 \pm .02$ & .03 & - & - & - & - & - & - \\
\hline
\end{tabular}

parameter was not a priori constrained (Seyfert \& Scorza 1996; Michard 1998), the logarithmic colour gradient of a bulge is not the same if measured in terms of minA or majA abscissae. We have here chosen to give bulge colour gradients $\Delta C / \log r$ in terms of the mean radius $r$. The results of the present attempt to measure bulge gradients, together with reference colours in the corresponding $r$ range, are given in Table 7 .

The measured reference colour for bulges are not much different of the isophotal colours at $r_{\mathrm{e}}$ given in the same table, because for this kind of objects, the $r_{\mathrm{e}}$ values do not fall much farther outside than the radii adopted here to define the bulge colours. The estimated bulge gradients are also similar to the isophotal radial gradients, because the measurement ranges often overlap, so that the gradients are bulge dominated. In a few favourable cases, i.e. NGC 3115, 4350, 5308, the bulge gradient is larger than the average one.

\subsubsection{Disk colours and gradients}

For most of the galaxies in the sample, there are extended stretches of the majA where the light is essentially due to the disk: such regions can be located from the bulge+disk analysis quoted above. An exception is NGC 3115 where the disk is nowhere much brighter than the spheroid. Disk colours and gradients have been measured wherever it seemed feasible, and the results are given in Table 8 .

The gradients are calculated against the majA $l$, so that they might be compared to various radial gradients of interest for disk galaxies. They cannot be compared to the mean isophotal gradients above without precautions, because of the variations of axis ratio in the range of measurement. It has been checked however that $\Delta C / \log l$ and $\Delta C / \log r$ are the same within errors, except in a few cases, where the later is larger in connection with a strong decrease of $q$ in the domain of measurement. It should also be stressed that the run of majA colours may show significant fluctuations associated with ring-lens structures (see below), making the evaluation of gradients somewhat unsecure.

We have compared the disk colour gradients $(D C G)$ of Table 8 to the bulge colour gradients $(B C G)$ of Table 7 (sometimes replaced by the isophotal gradients if deemed equivalent): it is found that disk gradients are systematically smaller. A numerical comparison give the following results:

. $B-V 8$ cases, mean $B C G: .10$, mean $B C G-D C G$ : $.05, \sigma: .04$ 
NGC4026 B-R t116 $0.75^{\prime \prime}$

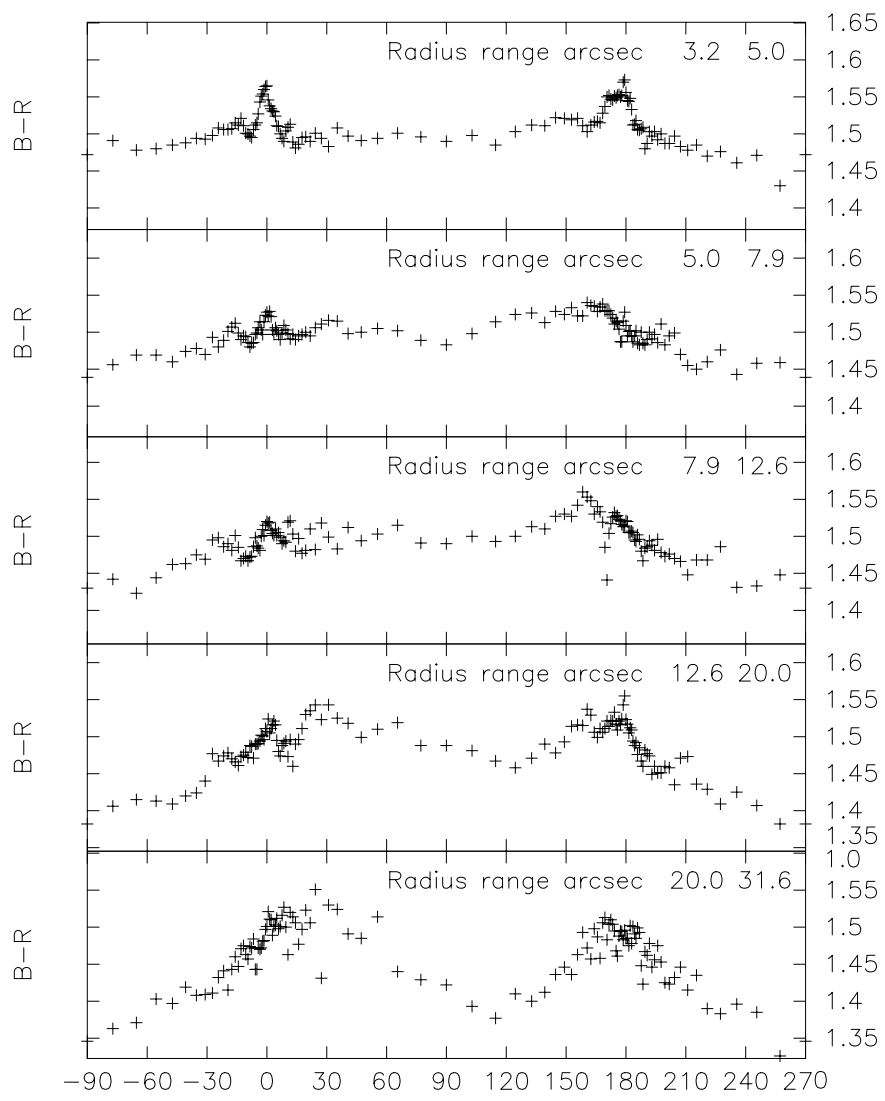

Fig. 1. Azimuthal $B-R$ colour profiles for NGC 4026 from TBL frames. Abscissae are polar angles in degrees, with the majA at 0 and 180 and the $\mathrm{E}$ min A at 90 . The colours are measured within ranges of isophotal radii given as labels to each curve. Interesting features are the very red narrow inner disk; the blueing of the minA regions in the outer isophotal "rings"; the colour asymmetry in the minA regions, the eastern one being redder, with definite red peak due to dust, near PA of 30 and $150^{\circ}$

. $V-I 7$ cases, mean $B C G$ : .035, mean $B C G-D C G$ : $.00, \sigma: .02$

. $B-R 8$ cases, mean $B C G$ : .11, mean $B C G-D C G$ : $.05, \sigma: .04$

. $U-V 6$ cases, mean $B C G: .24$, mean $B C G-D C G$ : $.13, \sigma: .04$.

Note that measurement errors provide a large part of the $\sigma$ values for the differences in gradient between the two components.

On the other hand, the reference colours quoted for bulge and disk in a given galaxy are very much alike, without systematic differences. Further examination will show that, if taken upon a given isophote, majA colours (disk dominated) may differ slightly from minA colours (bulge or envelope dominated).
NGC4026 Azimuthal profiles

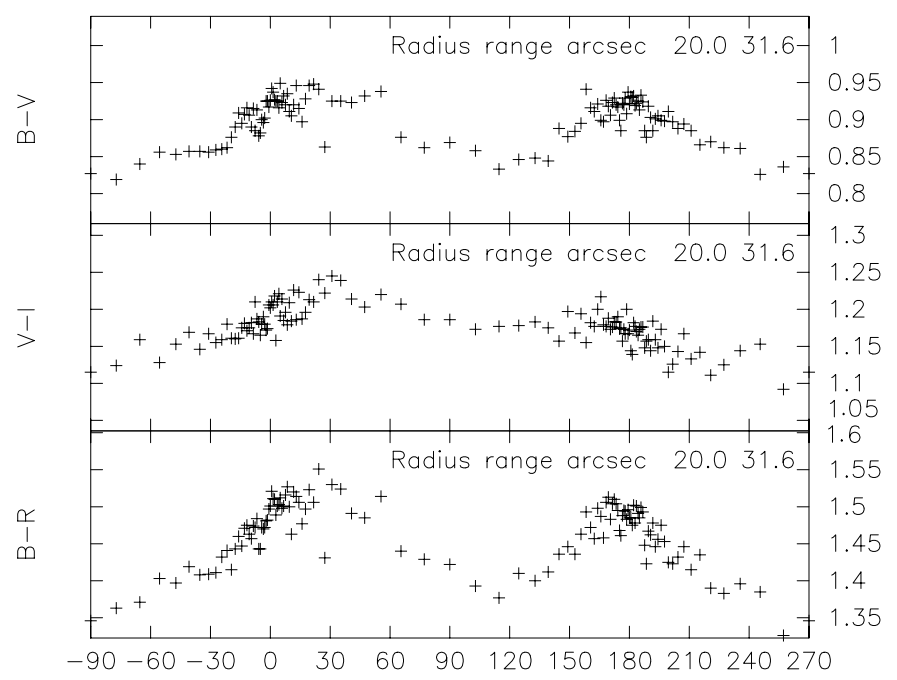

Fig. 2. Azimuthal colour profiles in NGC 4026 from TBL frames. This completes Fig. 11 by showing the profiles for the outermost measured range in three different colours. The colour contrast between the majA and the minA is minimum in $V-I$

Table 9. Measurements of the colour contrast of the dust lane in NGC 4036. Obs: Source of the data. Colour contrasts of the dust lane are averages of several points selected in $2 \mathrm{D}$ colour maps against an ad hoc background (see text). They are in magnitudes

\begin{tabular}{llllll}
\hline NGC & Obs. & $B-V$ & $V-I$ & $B-R$ & $U-V$ \\
\hline 4036 & TBL & 0.18 & 0.19 & 0.25 & - \\
\hline id & OHP & 0.15 & 0.11 & 0.21 & 0.24 \\
\hline
\end{tabular}

\subsection{Colour maps and dust patterns}

Maps of isochromes and isophotes are shown in Figs. A1 to A12 in the Appendix. Such maps are specially useful to recognize "dust patterns" and their geometries. These are of course well seen in objects listed in the subclass $\mathrm{SO}_{3}$ of the RSA, that is NGC 4036 and 5422, and less easily in the subclass $\mathrm{SO}_{2}$ as NGC 7332. Besides this, dust patterns are detected in the colour maps of several S0's. Small size but high contrast patterns are seen in NGC 3245 and 4111. More extended low contrast dust formations appear in the colour maps of NGC 2549 and 4026. The Table 9 gives a few measurements of the contrast of dust formations in NGC 4036. Here the measured points were identified on $2 \mathrm{D}$ colour maps, while the background was chosen as the average colour upon an isophotal contour outside the pattern. It should be noted that colour contrasts are distinctly smaller for the OHP frames, as a result of their poor seeing. In view of seeing effects, relative colour contrasts are more or less compatible with expectations for the same type of dust as the one in our Galaxy. 
Table 10. Selected measurements of mean colours and colour differences upon major and minor axes. A colon is appended to more uncertain values. OBS: source of observations. $r$ : Mean isophotal radius. $l$ and $s$ : true semi-major and - minor axes. $B-V$, etc.: mean isophotal colours. $\Delta C$ : colour difference, i.e. colour at the majA minus colour at the minA. Not: Notes. a) Inner disk of NGC 4026. b) NGC 4036: possible effect of dust pattern. c) NGC 4036: well outside dust pattern. d) NGC 4350: dusty disk? e) Inner disk of NGC 5308

\begin{tabular}{|c|c|c|c|c|c|c|c|c|c|c|c|c|c|}
\hline$\overline{\text { NGC }}$ & $\overline{\text { OBS }}$ & $r$ & $l$ & $s$ & $B-V$ & $\Delta C$ & $V-I$ & $\Delta C$ & $B-R$ & $\Delta C$ & $U-V$ & $\Delta C$ & Not \\
\hline 3098 & $\mathrm{CFH}$ & 16. & 37. & 6.9 & - & - & - & - & 1.38 & 0.10 & - & - & \\
\hline 4026 & TBL & 4.0 & 5.0 & 3.0 & 0.93 & .085 & 1.18 & .015 & 1.49 & .07 & - & - & $a$ \\
\hline id & id & 6.3 & 7.5 & 4.8 & 0.91 & .04 & 1.16 & .01 & 1.47 & .06 & - & - & - \\
\hline id & id & 10. & 13. & 7.1 & 0.91 & .05 & 1.15 & .01 & 1.46 & .055 & - & - & - \\
\hline id & id & 16. & 25. & 10. & 0.89 & .08 & 1.15 & .02 & 1.43 & .085 & - & - & - \\
\hline id & id & 25. & 55. & 14. & 0.86 & .09 & 1.14 & .04 & 1.42 & .125 & - & - & - \\
\hline 4026 & OHP & 10. & 13. & 7.1 & 0.92 & .02 & - & - & 1.48 & .03 & 1.45 & .09 & - \\
\hline id & id & 16. & 25. & 10. & 0.91 & .03 & - & - & 1.47 & .045 & 1.42 & .105 & - \\
\hline id & id & 25. & 55. & 14. & 0.87 & .05 & - & - & 1.45 & .06 & 1.36 & .155 & - \\
\hline id & id & 40. & 91. & 20 . & 0.84 & .09 & - & - & 1.45 & .05 & 1.32 & .21 & - \\
\hline 4036 & TBL & 16. & 25. & 10. & 0.93 & .11 & 1.17 & .07 & 1.48 & .13 & - & - & $\mathrm{b}$ \\
\hline id & id & 25. & 40. & 15. & 0.91 & .085 & 1.13 & .015 & 1.43 & .085 & - & - & $\mathrm{c}$ \\
\hline 4036 & OHP & 16. & 25. & 10. & 0.93 & .06 & 1.19 & .05 & 1.49 & .08 & 1.51 & .14 & $\mathrm{~b}$ \\
\hline id & id & 25. & 40. & 15. & 0.90 & .045 & 1.15 & .03 & 1.44 & .04 & 1.43 & .130 & $\mathrm{c}$ \\
\hline id & id & 40. & 65. & 25. & 0.92 & .015 & 1.13 & .01 & 1.43 & -.01 & 1.36 & .08 & - \\
\hline 4350 & $\overline{\mathrm{OHP}}$ & 10. & 16. & 6. & 0.93 & .03 & 1.22 & .04 & 1.50 & .04 & 1.43: & $.22:$ & $\bar{d}$ \\
\hline id & id & 16. & 29. & 9. & 0.92 & .05 & 1.19 & .08 & 1.47 & .06 & 1.39: & $.21:$ & d \\
\hline id & id & 25. & 46. & 14. & 0.89 & .04 & 1.17 & .09 & 1.44 & .10 & 1.28: & $.21:$ & $\mathrm{d}$ \\
\hline id & id & 40. & 64. & 25. & 0.83 & $.06:$ & 1.18 & .10 & 1.42 & .10 & - & - & \\
\hline 5308 & TBL & 4.0 & 5.1 & 2.8 & 0.90 & .070 & 1.18 & .040 & 1.45 & .095 & - & - & $\mathrm{e}$ \\
\hline id & id & 6.3 & 8.5 & 4.2 & 0.90 & .060 & 1.18 & .035 & 1.44 & .080 & - & - & - \\
\hline id & id & 10. & 20. & 5.8 & 0.89 & .085 & 1.18 & .040 & 1.43 & .095 & - & - & - \\
\hline id & id & 16. & 36. & 8.3 & 0.85 & .110 & 1.16 & .055 & 1.39 & .130 & - & - & - \\
\hline id & id & 25. & 56. & 12. & 0.81 & .160 & 1.13 & .055 & 1.35 & .170 & - & - & - \\
\hline
\end{tabular}

Besides obvious dust patterns, colour maps show possible dust concentration in the inner disk of such objects as NGC 3115, 4026, 5308, where the inner isochromes are much flatter than the isophotes. This phenomenon is further discussed below.

\subsection{Isochromes against isophotes}

The deviations of isochromes from isophotes have been studied mostly from many "azimuthal colour profiles": examples of such diagrams are shown in Figs. 1, 2 and 4. If the isochromes follow the isophotes, the azimuthal profiles are horizontal lines except for noise fluctuations. This is so for NGC 2549 or 7457 (with rather poor S/N ratio). In other cases various kinds of fluctuations occur, regular or not, and located either near the center of the object or at large $r$ values. These deviations are possibly due to diffferent physical causes, and are best described by simultaneously considering the 2D light and colour maps.

Features in the azimuthal profiles are classified as follows:
1. "Dust patterns", already discussed in the previous subsection, show up as red peaks in the azimuthal profiles, where the "measurement ring" intercepts such a feature;

2. "Diffuse dust in disks" may be present without forming well defined patterns, due to an unfavourable projection, or to a diffuse large scale distribution. The dust layer may then be detected by a reddening of the majA as compared to adjoining regions. For a favourable range of inclinations, it may also produce an asymmetry in light and colour along the minA, as the extinction and reddening are larger on one side of the bulge than the other. A discussion of this phenomenon may be found in MS93. From these criteria, dust is present in NGC 4026, 4350, 5422. From the first criterion alone, dust might also be present in the innermost range of the disk of NGC 3115, up to a majA length $a=5$ arcsec, and in part of the disk of NGC 5308, up to $a=15$. A red central disklet is also seen in NGC 4026, according to Fig. 1. This criterion is however not unambiguous, because the disk might well be 


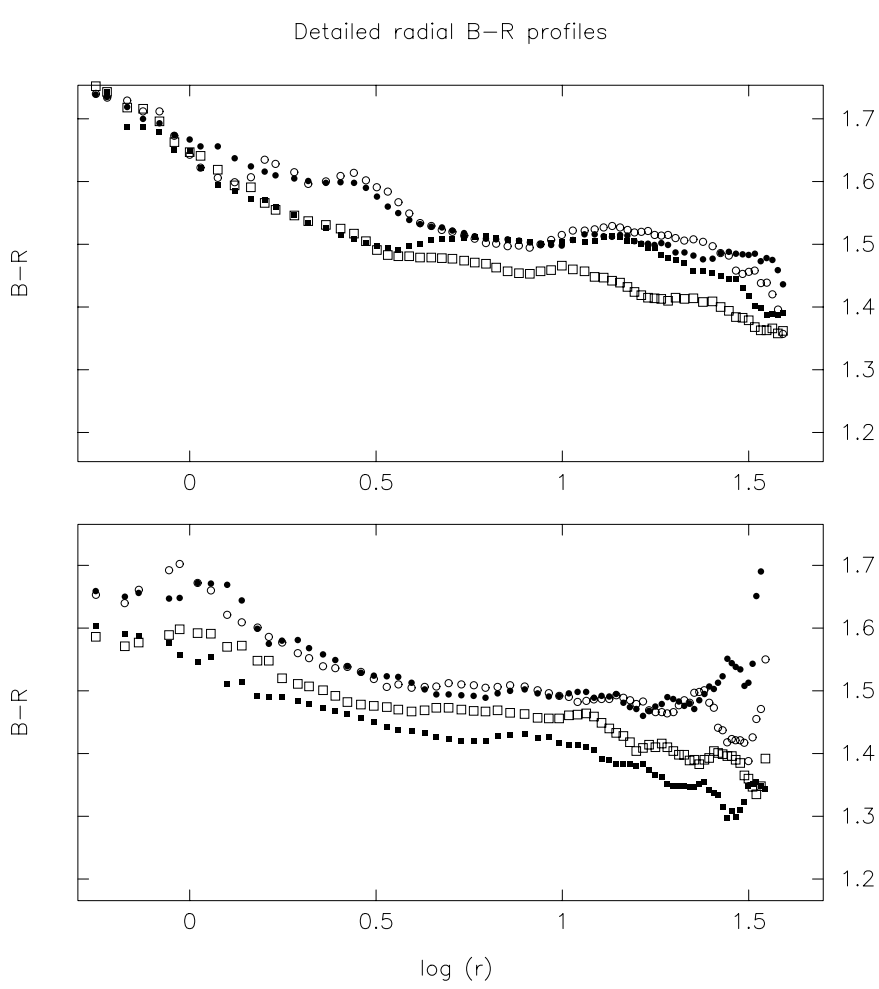

Fig. 3. Radial $B-R$ colour profiles in NGC 4026 and 5308 from TBL frames. Abscissae: Mean isophotal radius $r$ in arcsec and logarithmic scale. The colours of the 4 halves of each axes are shown separately. Upper frame: NGC 4026, PA $=179^{\circ}$. Full dots: South majA. Small circles: North majA. Black squares: East $\min A$. White squares: West $\min A$. The $\min A$ becomes bluer outwards than the majA, much more so for the western side, as the eastern one is affected by dust (see also Fig. 14). Lower frame: NGC 5308, PA $=59^{\circ}$. The various symbols then relate to orientations $120^{\circ}$ clockwise from the ones above. The majA is redder everywhere, due to a thin red disk at small $r$ and a blue bulge at large $r$. There is no minA asymmetry in this case

locally redder than the adjoining regions from a difference in their stellar populations. Indeed we find that the inner "red disks" of NGC 4026 and 5308 have less colour contrast in $V-I$ than $B-V$ : this is not in line with the dust layer explanation... which however cannot be ruled out due to the effects of seeing upon these minute features;

3. From azimuthal colour profiles a thin disk bluer than its surrounding occurs in NGC 3098, inwards of a radius of about $r_{\mathrm{e}}$, or 8 arcsec. This is also the case in NGC 3115, roughly in the range $15<r_{\mathrm{e}}<30$. The blue disk of NGC 3098 is washed out in our poor resolution OHP data. The one of NGC 3115 is barely seen at the limit of our small field CFHT data. This phenomenon is presented in Fig. 4;

4. "Blueing of the outer spheroidal component" of S0's is expected by analogy with ellipticals. Indeed in many of the azimuthal colour distribution, the outer measured isophotal rings show a large colour contrast between

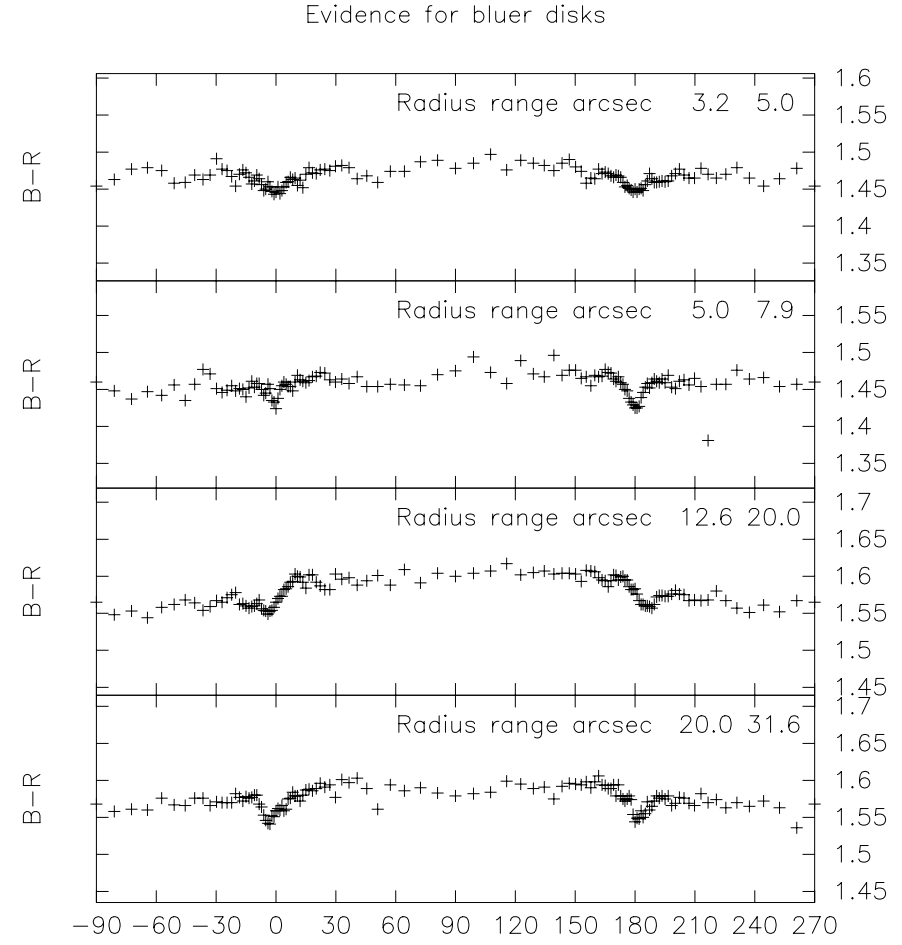

Fig. 4. Azimuthal colour profiles through the inner part of NGC 3098 (upper curves), and the medium part of NGC 3115 (lower curves). Abscissae are polar angles in degrees, with the majA at 0 and 180 . The colours are measured within ranges of isophotal radii given as labels to each curve. A narrow bluer region occurs along the majA

minor and major axes regions, the earlier ones being bluer. This is clear in our azimuthal colour profiles for NGC 3098, 4026, 4036, 4111, 4350, 5308, 5422, and is also marginally seen in others. It is easily checked that the minA regions become bluer with increasing radius, while the majA regions stay at nearly the same redder colour (see Fig. 3 for examples). It is therefore proposed that this feature of the azimuthal colour profiles is due to a colour gradient (similar to to the one observed in E-type galaxies that is a blueing towards larger radii), occuring in galaxian components other than the disk, that is the bulge itself and an outer envelope (sheroidal halo or thick disk), which may or not be considered as an extension of the bulge. It is suggestive that the colour contrast here discussed is minimum in $V-I$ and maximum in $U-V$, exactly like the radial colour gradients discussed above.

In Table 10 are collected a number of results about the colour differences between the minor and major axes in our sample galaxies, as a function of isophotal radius and colour. As these differences are very sensitive to seeing, in the case of near edge-on galaxies, results for our OHP data are separated from the higher resolution CFHT and TBL data (and eventually discarded, if deemed too much affected by the poor PSF's). These data have been recovered from azimuthal colour profiles similar to Figs. 1 and 


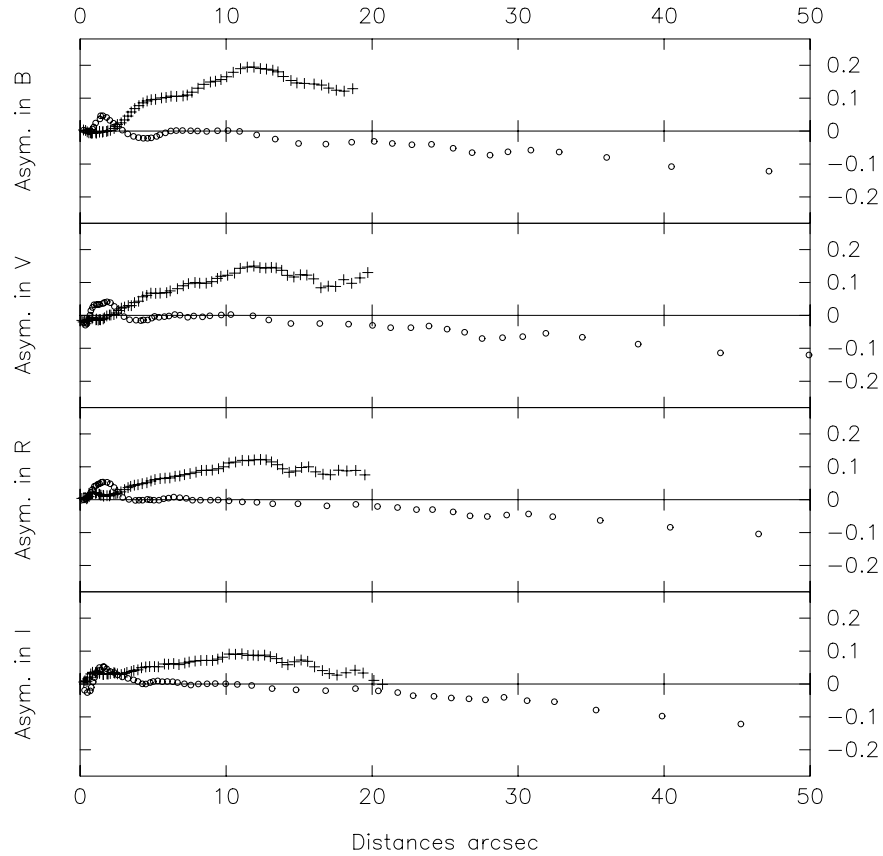

Fig. 5. Asymmetries in the $\mathrm{SuBr}$ distributions along the two axis of NGC 4026 from TBL frames. Abscissae: Radial distances in arcsec. Ordinates: Differences in magnitudes between the two axes, with circles for the majA and crosses for the $\min A$. There is no colour effect in the asymmetry along the majA, while the asymmetries along the minA change regularly with the colour: the E side is less bright and redder, due to dust in the disk of the object

2. Depending upon the studied object and radius range, the majA minus minA colour difference may be due either to dust concentration in the disk or to blueing of the bulge component, or both. Our guesses as regards the relevant effect are indicated below, in the descriptions of each object.

As is the case for ellipticals, one may wonder if the suggested colour gradients in the bulge of S0's may be explained by the advocated population gradients (see a review in Wyse et al. 1997), or if a concentration of diffuse dust towards the center has to be invoked, as in the theoretical models of Witt et al. (1992), and in the applications by Goudfrooij \& de Jong (1995), or Wise \& Silva (1996). It may be added that, in the case of S0's, dust concentration in the disk is likely to play a role in colour distributions, not only in the inner part of several objects as discussed above, but also in their outer radial range. The situation is expected to be rather complicated, since we deal with galaxies built from at least two components, i.e. bulge and disk, and two sources of colour variations, i.e. populations changes and dust occurence.

\subsection{The minor axis asymmetries}

The phenomenon described in MS93, and tentatively attributed to rather thin, but extensive, layers of dust in
Table 11. Measurements of the minor axis asymmetry. Obs: source of observations. $s$ : distance along the minA where the asymmetry is measured in arcsec. For NGC 5422 the $B$ measurement is from an OHP frame

\begin{tabular}{llllllll}
\hline NGC & Obs. & $s$ & $U$ & $B$ & $V$ & $R$ & $I$ \\
\hline 4026 & TBL & 12 & - & 0.18 & 0.15 & 0.11 & 0.08 \\
id & OHP & id & 0.20 & 0.11 & 0.09 & 0.08 & - \\
4036 & TBL & 25 & - & 0.14 & 0.13 & 0.11 & 0.08 \\
id & OHP & id & 0.12 & 0.13 & 0.11 & 0.07 & $0.08:$ \\
4350 & OHP & 12 & 0.10 & 0.10 & 0.08 & 0.08 & 0.05 \\
5422 & TBL & 15 & - & 0.20 & 0.13 & 0.09 & 0.03 \\
\hline
\end{tabular}

S0 disks, has been reconsidered from the present higher resolution data. A clear cut case is NGC 4026, where the asymmetries are shown in Figs. 5, in 4 bands from TBL data, and display the expected colour effect.

Table 11 gives some new measurements of the minA asymmetry. It is interesting that the measured asymmetries are smaller for OHP frames than for TBL frames (cases of NGC 4026 and 4036) probably as a seeing effect. It has been found however that in some cases OHP frames of edge-on galaxies display large minA asymmetries due to an elongated and asymmetric PSF!

MS93 emphasized that the data at hand did not allow to distinghuish between extensive continuous layers of dust, as postulated in their attempted modeling, or systems of dust clouds. The high resolution colour maps obtained for NGC 4026 and 5422 suggest that some structure is present in the disk of these objects, notwithstanding the unfavourable projection.

\section{Notes on individual galaxies}

The notes about the morphology of each object are taken from the results in MM93, completed from the study of the present high resolution frames. Use has also been made of the analysis in terms of bulge and disk components published by Michard (1998) (M 98) for most objects of the present sample. This same analysis has been performed for the others using the techniques in M 98. The notes about the colour distributions are from the present study.

1. NGC 2549. This object contains a roundish boxy bulge, and an extended disk, the dominant component at $a=20^{\prime \prime}$ and farther. Envelope class: thD. From observables and analysis in M 98, the disk present a ring structure with humps near 6.5 and $33^{\prime \prime}$. Associated small fluctuations of the $\mathrm{PA}$ and $f_{4}$ parameters may suggest some trace of spiral structure (?). The $q$ ratio of the disk component according to M 98 leads to an inclination larger than $80^{\circ}$.

The radial colour distributions in Fig. A1 are "regular", with rather flat $V-I$ and sharply decreasing $U-V$. The azimuthal colour profiles are flat, showing the isochromes and isophotes to be nearly coincident. 


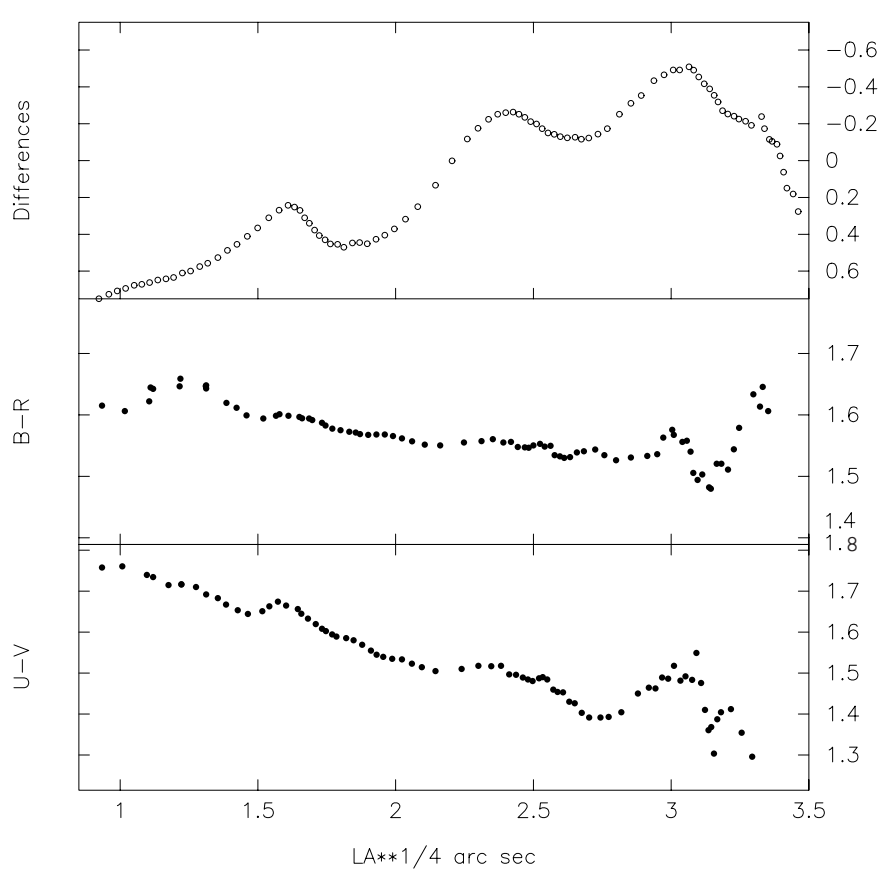

Fig. 6. Possible colour changes associated with rings in NGC 2549. Abscissae: majA central distances in arcsec and $r^{1 / 4}$ scale. Ordinates: Upper frame: Differences in mag between a $B$ majA $\mathrm{SuBr}$ profile and a least square fitted $r^{1 / 4}$ law: this strongly emphasizes local ring features. Middle frame: $B-R$ majA profile. Lower frame: $U-V$ majA profile. There is some correspondence between the ring features and humps in the colour profiles, particularly in $U-V$

The colour map of Fig. A1 shows a low contrast central pattern, with a thin arclet (dust?) in coincidence with an isophotal contour. An interesting property of this galaxy lies in the subtle changes of colour associated with the ring structure, which is demonstrated in Fig. 6;

2. NGC 3098. It presents a boxy bulge and a disk mixed in a spheroidal envelope. The disk is dominant at $a=6^{\prime \prime}$ and farther. Envelope class: thD. There is an inner disk, giving a fist $\epsilon$ maximum at $a=1.2^{\prime \prime}$, and a low contrast ring with the hump near $a=9^{\prime \prime}$. The $q$ ratio of the disk component according to M 98 leads to an inclination larger than $84^{\circ}$;

The radial colour distributions in Fig. 1 are "regular", while the colours are slightly bluer than average. No evidence for dust is seen. The CFHT frames show the majA to be bluer than surroundings for $a<10^{\prime \prime}$ (see Fig. 4), that is in the ring region. Farther out the reverse situation occurs (see Table 10), possibly due to an outwards blueing of the spheroidal component, compared to a disk with small colour gradient;

3. NGC 3115. The large bulge dominates the $\mathrm{SuBr}$, except where the low contrast ring shows up, around $a=18^{\prime \prime}$. Nieto et al. (1991) noted the inner "decoupled" disk inside $a=5^{\prime \prime}$. The main disk exhibits a cut-off near $a=90^{\prime \prime}$, so that the envelope is in class
spH. The $q$ ratio of the disk component according to M 98 leads to an inclination larger than $82^{\circ}$.

The radial colour distributions are surprisingly flat. We note that the object is difficult, too large for the field of the available OHP observations. Silva et al. (1989), found the majA slightly bluer than surroundings. From an analysis in terms of a bulge and disk components, they conclude that the disk population is bluer by 0.5 in $B-I$ than the bulge one, a conclusion very dependent upon their model. Our CFHT frames show the inner disk to be redder (see Fig. A3), while farther out the majA becomes bluer than surroundings, (see Fig. 4) in qualitative agreement with Silva et al. observations. A bulge+disk analysis according to the techniques in M 98, suggests that the $B-R$ differences between the two component remain small. The disk is found redder near $a=3^{\prime \prime}$ (inner disk) and bluer for $a>15^{\prime \prime}$ (main disk). The only evidence for dust in NGC 3115 is in the red inner disk, but this might be a population effect.

This nearby galaxy has been much studied spectroscopically: see the survey by Fischer (1996), and the discussion of a central black hole by Kormendy et al. (1996);

4. NGC 3245. This galaxy has a modest bulge and a strongly dominating disk. There is a double ring structure with maxima near $a=5^{\prime \prime}$ and $a=15^{\prime \prime}$. Envelope class: exD. The $q$ ratio of the disk component according to M 98 leads to an inclination of $62^{\circ}$.

The radial colour distributions in Fig. A4 are "regular". The relatively strong reddening towrds the center is due to a high contrast dust pattern within $3-4^{\prime \prime}$ from center (see map in A4). This in turn contains a blue feature, 1 " SE from center. Is it a "hole" in the dust, or a region of relatively recent star formation?

5. NGC 4026. This object has a strongly dominant disk extending out of a boxy bulge. There is again a minute inner disk giving a first peak of the $\epsilon$ profile at $a=4.5^{\prime \prime}$. The ring produces a hump in $\mathrm{SuBr}$ around $a=19^{\prime \prime}$. Envelope class: thD. An unpublished bulge+disk analysis with the techniques of M 98 leads to an inclination larger than $80^{\circ}$.

The radial colours (see Fig. A5) are "regular". A slightly redder hump in all colour curves appears at $r=12^{\prime \prime}$, or a majA of $a=19^{\prime \prime}$, which put it in coincidence with the ring. According to the high resolution map of Fig. A5, the "inner disk" is redder than surroundings: this reddening is found to be less in $V-I$ than $B-V$, which may rule out an explanation in terms of a dust concentration. NGC 4026 is not symmetric, the eastern side of the bulge being less bright and redder than the western (Figs. 1 and 5), due to rather thin dust throughout the disk, and/or a system of dust features. Finally, this galaxy presents a remarkable contrast between the majA and minA colours far from center (Figs. 1, 2 and Table 10), a phenomenon we 
have ascribed to an outwards blueing of the spheroidal component as compared to the disk;

6. NGC 4036. Again this object has a dominant disk. The morphology in the inner radius range is affected by a major dust pattern mostly along the majA. A low contrast ring may be present with maximum near $a=28^{\prime \prime}$. An unpublished bulge+disk analysis with the techniques of M 98 leads to an inclination of $73^{\circ}$. Envelope: exD class.

An outstanding dust pattern is present (Fig. A6): three lanes of irregular structure can be recognized, with a geometry suggesting that the dust lies in the disk plane. The colour excess locally reaches $E(B-V)=$ 0.18 against background. Besides this, the object shows the min A asymmetry, with the $\mathrm{S}$ side redder and less bright, farther out than the lanes. The phenomenon described as "blueing of the outer bulge" is also present (Table 10);

7. NGC 4111. In the central region is seen the long ago described "peanut bulge" (Tsikoudi 1980). There is also an inner disk, giving a first $\epsilon$ maximum near $a=5^{\prime \prime}$, and a ring showing up near $a=22^{\prime \prime}$. Envelope class: thD.

There is a small but high contrast central dust pattern, elongated along both galaxian axes. The map of Fig. A7 suggests that the peanut bulge may result from extinction by the overlying dust. Indeed it has been verified from the original frames, that a dust lane crosses the bulge along the minA, slightly $\mathrm{S}$ from center, and shows much less contrast in $R$ than in $B$ (no $I$ frame is available). It is our guess that the peanut bulge would not be seen in the $K$ band. The object is very blue compared to others in the sample, specially in the disk, away from the central dusty bulge, and in the $U-V$ colour (Table 8): its stellar population is probably younger than average. The outer isophotes show, to a rather small extent, the blueing of the minA relative to majA noted for several other objects;

8. NGC 4350. This S0 is typical of galaxies with an spH type envelope, as the outer axis ratio increases to about 0.8. According to the analysis in M 98, the disk suffers an outer cut-off starting near $a=50^{\prime \prime}$, while the spheroidal component increases well above an $r^{1 / 4}$ extrapolation of the inner bulge. The inclination may be $77^{\circ}$ or larger.

The radial colour distributions (Fig. A8) show a red central peak and a gradient above average in all colours. There is evidence in our data for a reddening of the majA compared to surroundings (see $B-R$ map), and for minA asymmetry, the $E$ side being brighter and bluer. A concentration of dust in the disk is proposed;

9. NGC 5308. As in several other S0's, there is an inner disk with peak $\mathrm{SuBr}$ near $a=7^{\prime \prime}$. The bulge is rather boxy, and the envelope of the thD class. The analysis in M 98 points to a disk inclination of $83^{\circ}$, if infinitely thin, but it should be larger and close to $90^{\circ}$.

The radial colours are standard, except for a prominent red peak inwards of $r=2.5^{\prime \prime}$. This may be associated with the reddening of the inner disk against surroundings (Fig. A9, Table 10). Besides the object displays a strong reddening of the outer majA, again ascribed to the outwards blueing of the spheroidal component;

10. NGC 5422. This galaxy has a strong bulge, clearly brighter on the $\mathrm{W}$ side than on the $\mathrm{E}$ one. Again there is an inner disk, with first $\epsilon$ maximum near $a=6^{\prime \prime}$, and a ring with maximum contrast near $a=23^{\prime \prime}$. Envelope: thD class. From the M 98 analysis, the inclination is $81^{\circ}$, or larger.

The object displays high contrast dust markings nearly along the majA, but slightly displaced westwards. On the other hand there is a general reddening of the eastern side due to dust in the disk. This also produces a well defined minA asymmetry (Table 11);

11. NGC 7332. This object presents a boxy bulge, an inner disk with the first $\epsilon$ maximum near $a=4^{\prime \prime}$, and a ring with maximum contrast near $a=28^{\prime \prime}$. Envelope class: thD. A spectroscopic study of this object is due to Fisher et al. (1994).

Our radial $B-R$ profile for NGC 7332 are rather uncertain. The object displays a large dust pattern of moderate contrast (Fig. A11). As for NGC 4111, the disk is rather blue;

12. NGC 7457. This galaxy is less inclined than other sample objects, with a minimum axis ratio of 0.52 . Because the Carter's $e_{4}, e_{6}$ coefficients fluctuate around zero, it is believed that one of the two components, most probably the disk, dominates the other. Envelope class: uncertain. HST observations were published by Lauer et al. (1991).

The colours of this objects are quite flat, except for a very small central red peak (Fig. A12). They are also bluer than for most other S0's.

\section{Conclusions}

The present observations of colour distributions in $12 \mathrm{~S} 0$ galaxies, most of these nearly edge-on, suggest the following conclusions:

1. Radial isophotal colour distributions show some outwards blueing as in E type galaxies. The relative gradients in the 4 colour indices $B-V, V-I, B-R$, $U-V$, are not proportional to relative dust reddening coefficients, the $V-I$ gradient beeing quite small and the $U-V$ one quite large: apparently dust cannot be responsible for such gradients. When it is feasible to measure both the bulge and disk colour gradients the first one is steeper;

2. Optical dust is detected in 10 of the studied objects out of 12 , either from definite dust patterns 
(NGC 2549, 3245, 4026, 4036, 4111, 5422, 7332), or from indirect but ambiguous evidence. It is observed that the "peanut bulge" of NGC 4111 possibly results from the central dust pattern in this object;

3. Dust is likely to be present, without forming clear patterns, in the disk of NGC 4350, 5308, and perhaps also in the inner "decoupled disk" of NGC 3115. In these 3 cases the evidence comes from reddening of the majA as compared to its surroundings, along isophotal contours: it remains ambiguous however, since population differences might give similar observational effects. Checks from the use of various colour indices are not as yet conclusive;

4. A disk slightly bluer than the adjoining regions is found in NGC 3098 and NGC 3115, but only at $a>15^{\prime \prime}$ or so in this later case;

5. For a number of objects, the minA regions along a given isophote, become bluer than the majA tips. This might perhaps be explained in terms of spheroid + disk models, assuming a a strong outwards blue gradient in the spheroid, against a flater colour disk. By "spheroid" we here mean the superposition of the bulge itself and a thD or $\mathrm{spH}$ envelope, to use the classification scheme of MM93, much less flat than the disk. In principle some dust concentration in the disk might also provide an explanation, but our evidence from the comparison of the $B-V$ and $V-I$ colour indices does not support it;

6. Some correlations are found between the ring struture and the colour along the majA in NGC 2549 and 4026, more pronounced in $U-V$ : there a blueing of the colour profile coincides with the upwards "humps" in the $\mathrm{SuBr}$ profiles.

\section{Appendix}

The Appendix presents graphical data for each galaxy. These are not corrected for galactic extinction and $\mathrm{K}$ effect, contrary to the tabulated data. Each figure contains:

1. a map of isochromes (full line) and isophotes (doted), with coordinates in arcsec, the origin beeing at the galaxian center. The field may vary from map to map. The caption to each figure contains the direction of the North (the East is always 90 degrees counterclockwise), the levels and steps for the isochromes and isophotes. The maps are from the high resolution observations, CFHT or TBL, except for NGC 4350 where OHP frames had to be prefered;
2. a graph of the radial distributions of $R$ isophotal $\mathrm{SuBr}$ and available colours. Abscissae: $\log$ of isophotal mean radius $r$ in arcsec. Ordinates: magnitudes. OHP low resolution data are shown as circles, high resolution data as heavy dots ( $R$ curve) or stars (colour curves). Errors bars as evaluated in 3.4 are shown at $6 r$ values cooresponding to $\mu_{V}=19-24$.

\section{References}

Balcells M., Peletier R.F., 1994, AJ 107, 135 (BP94)

Bothun G.D., Gregg M.D., 1990, ApJ 350, 73

Fisher D., Illingworth G., Franx M., 1994, AJ 107, 160

Fisher D., Franx M., Illingworth G., 1996, ApJ 459, 110

Goudfrooij P., de Jong T., 1995, A\&A 298, 784

Kormendy J., Bender R., Richstone D., et al., 1996, ApJ 459, L57

Lauer T.R., Faber S.M., Holtzmann J.A., et al., 1991, ApJ 369, L41

Longo G., de Vaucouleurs A., 1983, A General Catalogue of Photoelectric Magnitudes and Colours in the $U B V$ System of 3578 Galaxies brighter than the 16th $V$-magnitude, 1936-1982. The Univ. of Texas Publ.

Longo G., de Vaucouleurs A., 1985, Supplement to the General Catalogue of Photoelectric Magnitudes and Colors in the $U B V$ System. The Univ. of Texas Publ.

Michard R., Marchal J., 1993, A\&AS 98, 29 (MM93)

Michard R., Marchal J., 1994, A\&AS 105, 481 (MM94)

Michard R., 1996, A\&AS 117, 583

Michard R., 1998, A\&AS 127, 299 (M 98)

Michard R., 1999, A\&AS 137, 245 (Paper IV)

Michard R., Simien F., 1993, A\&A 274, L25 (MS93)

Nieto J.L., Bender R., Arnaud J., et al., 1991a, A\&A 244, L25

Peletier R.F., Balcells M., 1996, AJ 111, 2238

Peletier R.F., Balcells M., 1997, NewA 1, 349

Sandage A., 1961, The Hubble Atlas of Galaxies, Carnegie Inst. of Washington (RSA)

Seyfert W., Scorza C., 1996, A\&A 310, 75

Silva D.R., Boroson T.A., Thompson I.B., Jedrzejewski R.I., 1989, AJ 98, 131

Tsikoudi V., 1980, ApJS 43, 365

Longo G., de Vaucouleurs A., 1983, A General Catalogue of Photoelectric Magnitudes and Colours in the $U B V$ System of 3578 Galaxies brighter than the 16th $V$-magnitude, 1936-1982. The Univ. of Texas Publ.

Longo G., de Vaucouleurs A., 1985, Supplement to the General Catalogue of Photoelectric Magnitudes and Colors in the $U B V$ System. The Univ. of Texas Publ.

de Vaucouleurs G., de Vaucouleurs A., Corwin H.G.Jr., et al., 1991, Third Reference Catalogue of Bright Galaxies. Springer Verlag, New-York

Wise M.W., Silva D.R., 1996, ApJ 461, 155

Witt A., Thronson H.A. Jr., Capuano J.M., 1992, ApJ 393, 611

Wyse R.F.G., Gilmore G., Franx M., 1997, ARA\&A 35, 617 

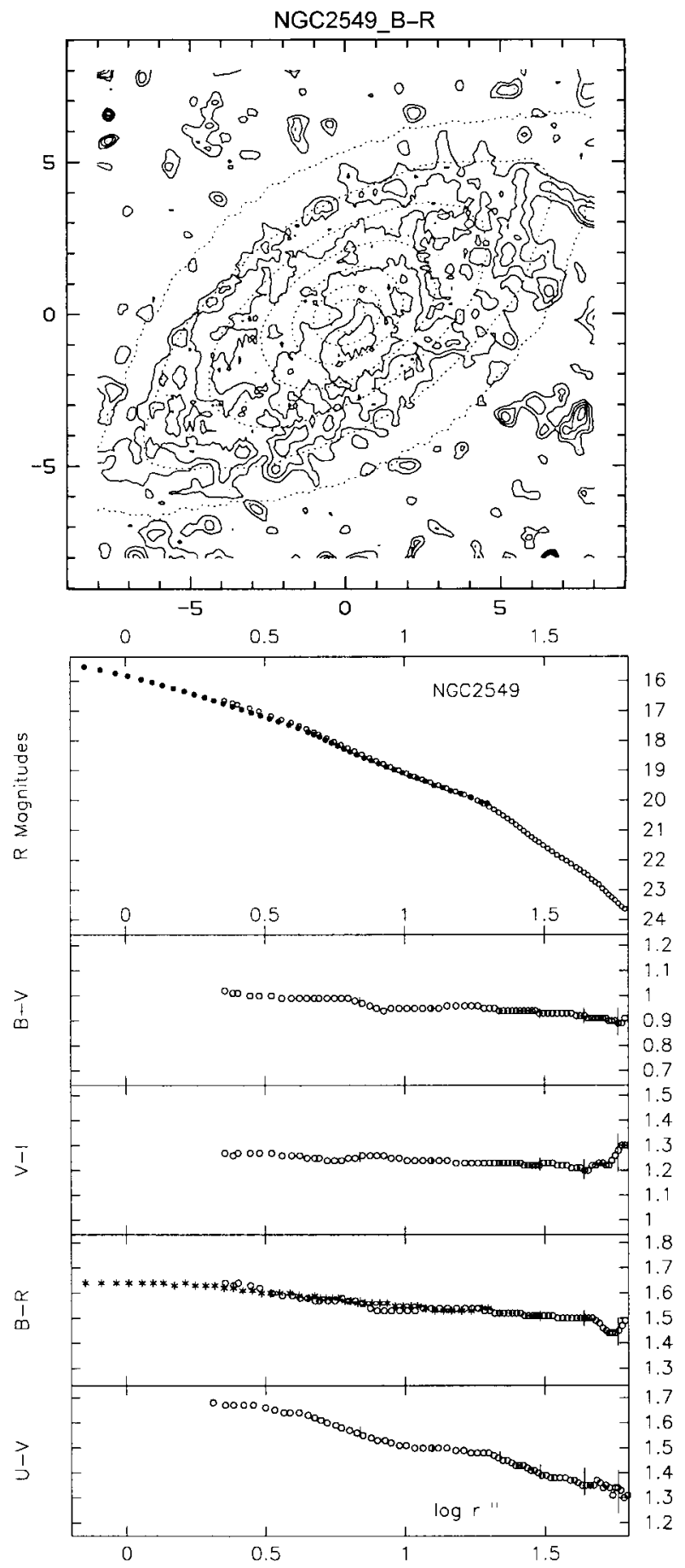

Fig. A1. NGC 2549: $B-R$ isochromes and $B$ isophotes from CFHT frames. Isochromes $1.60-1.675$ (0.025). Isophotes $17-20(0.5) . N$ at 8 o'clock. There is a low contrast dust pattern, with the peak red colour somewhat displaced from the galaxian center, and a thin lane along part of the 19.5 isophote (see also Fig. 6)
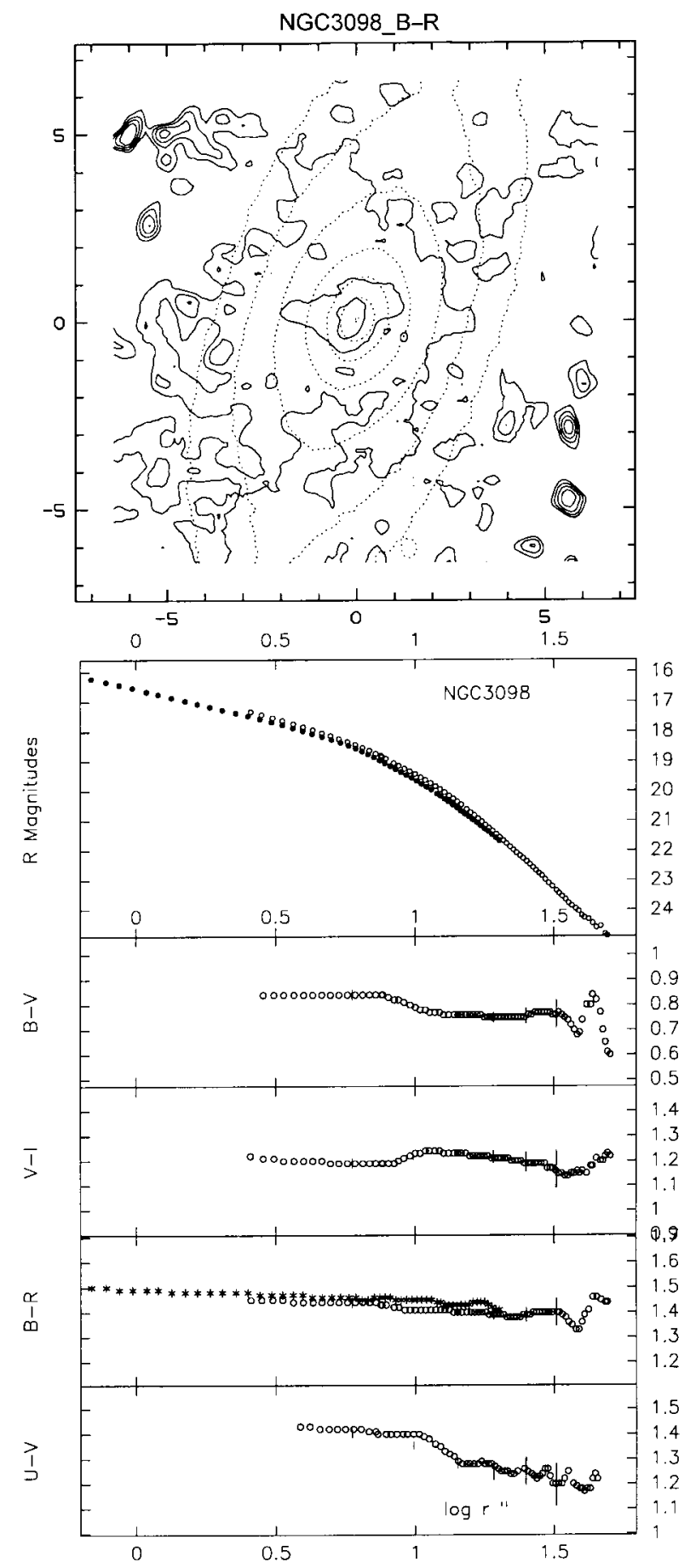

Fig. A2. NGC 3098: $B-R$ isochromes and $B$ isophotes from CFHT frames. Isochromes $1.475-1.55(0.025)$. Isophotes $17-20(0.5) . N$ at 930 o'clock. The colour distribution is rather flat, except for a modest central red peak, and evidence for the bluer disk at the SE majA. (see also Fig. 4). Poor $B-V$, $V-I$ colour profiles 


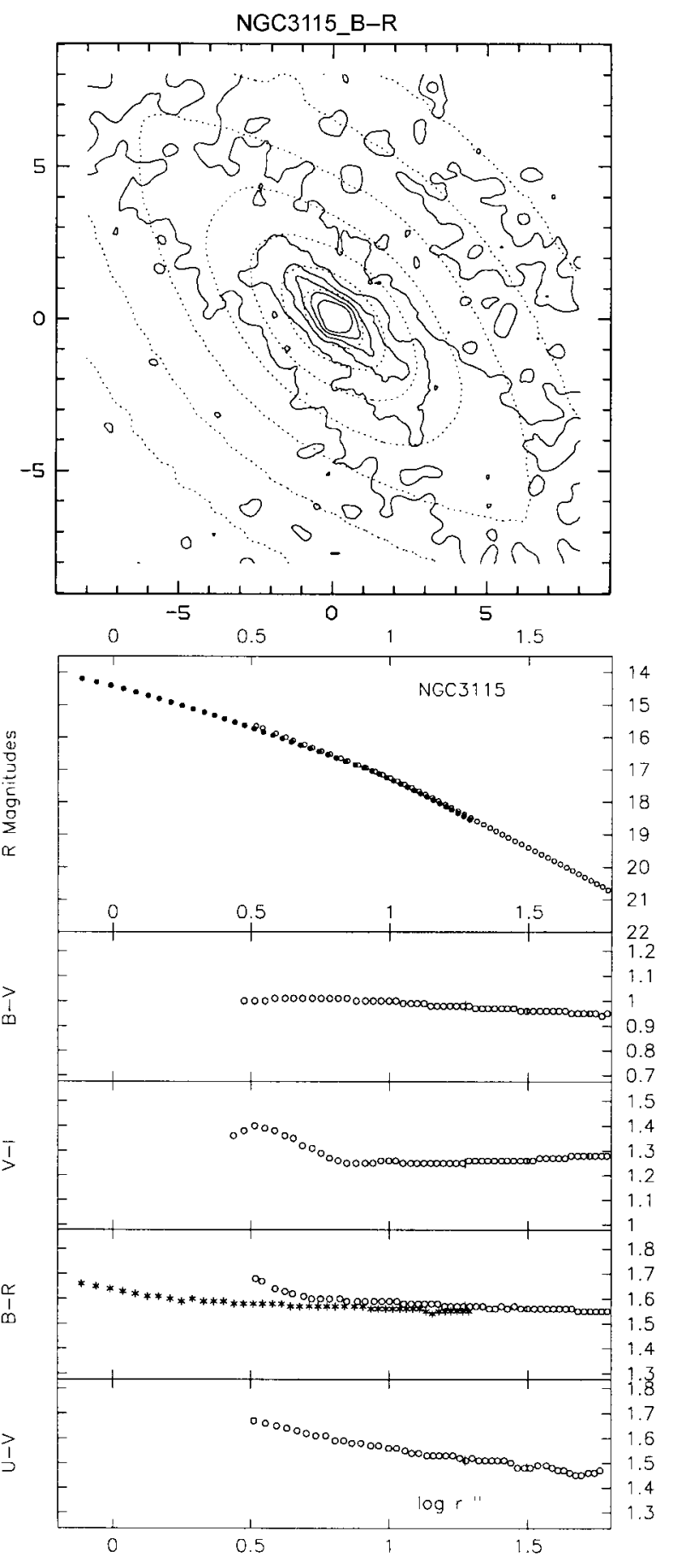

Fig. A3. NGC 3115: $B-R$ isochromes and $B$ isophotes from CFHT frames. Isochromes $1.55-1.675$ (0.025). Isophotes $16-19$ (0.5). $N$ at 9 o'clock. The innermost "decoupled disk", first noted by Nieto et al. (1991), is very red, possibly due to dust concentration there. Poor $V-I$ colour profile

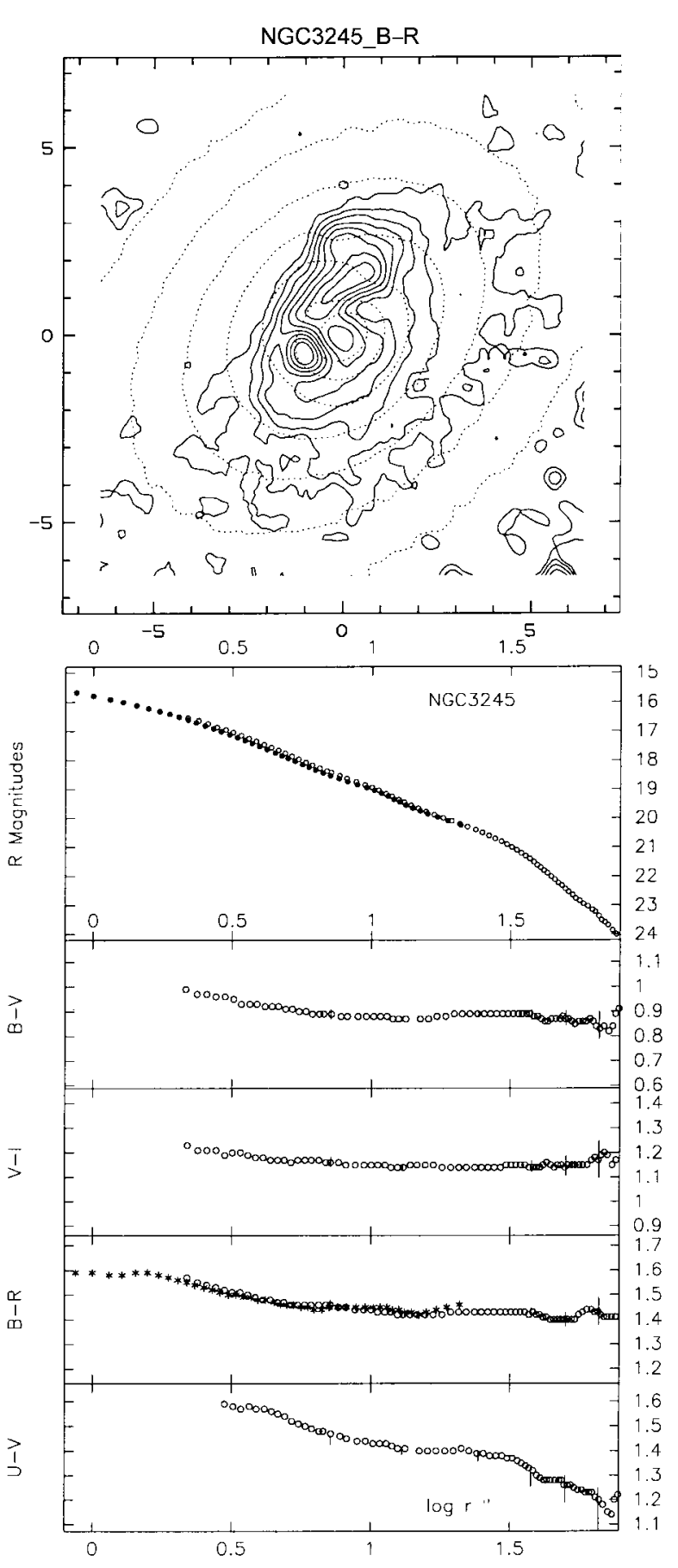

Fig. A4. NGC 3245: $B-R$ isochromes and $B$ isophotes from CFHT frames. Isochromes $1.50-1.70$ (0.025). Isophotes $17-20$ (0.5). $N$ at 730 o'clock. This object shows a high contrast central dust pattern. Note that the sharp feature $1.2 \operatorname{arcsec} N$ from center is bluer than its surroundings 


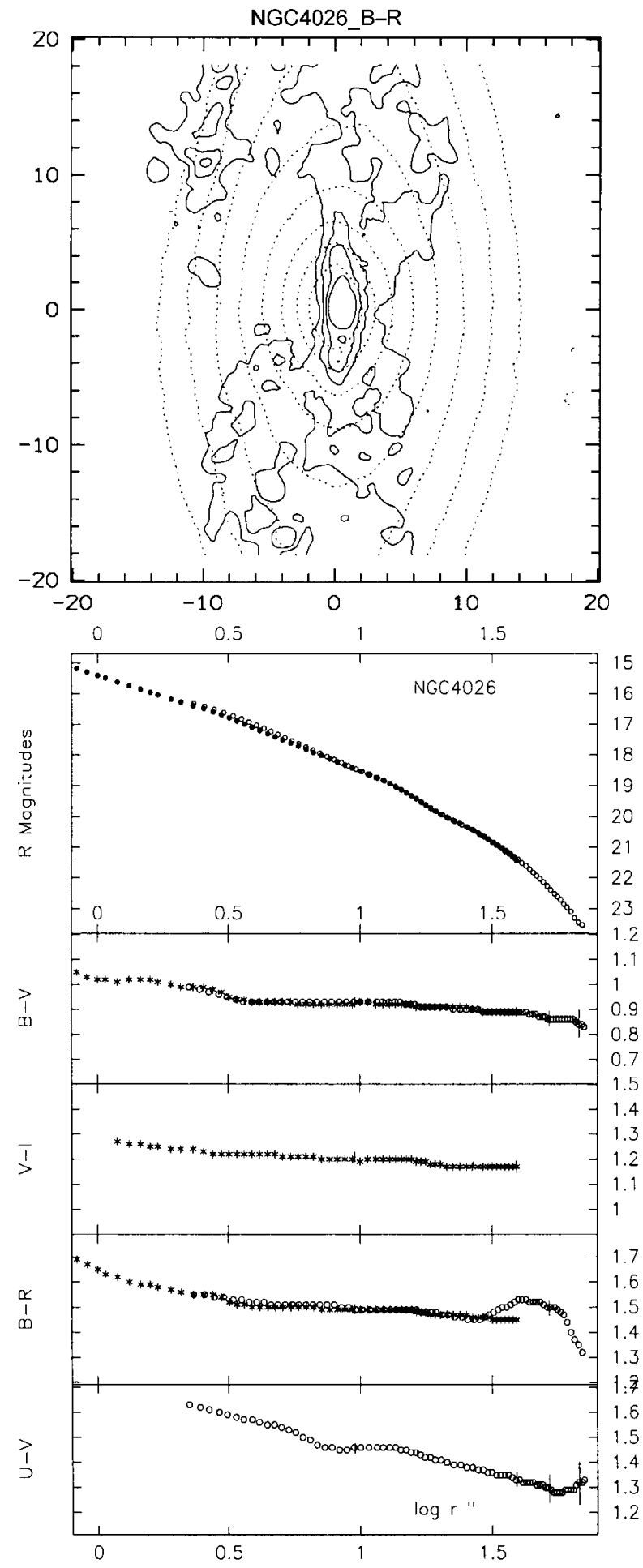

Fig. A5. NGC 4026: $B-R$ isochromes and $B$ isophotes from TBL frames. Isochromes $1.50-1.62(0.04)$. Isophotes $18-21.5$ (0.5). $N$ at the top. There is an inner thin red disk. This galaxy features the minA asymmetry in light and colour, the $E$ side being redder and less bright (see also Fig. 5)

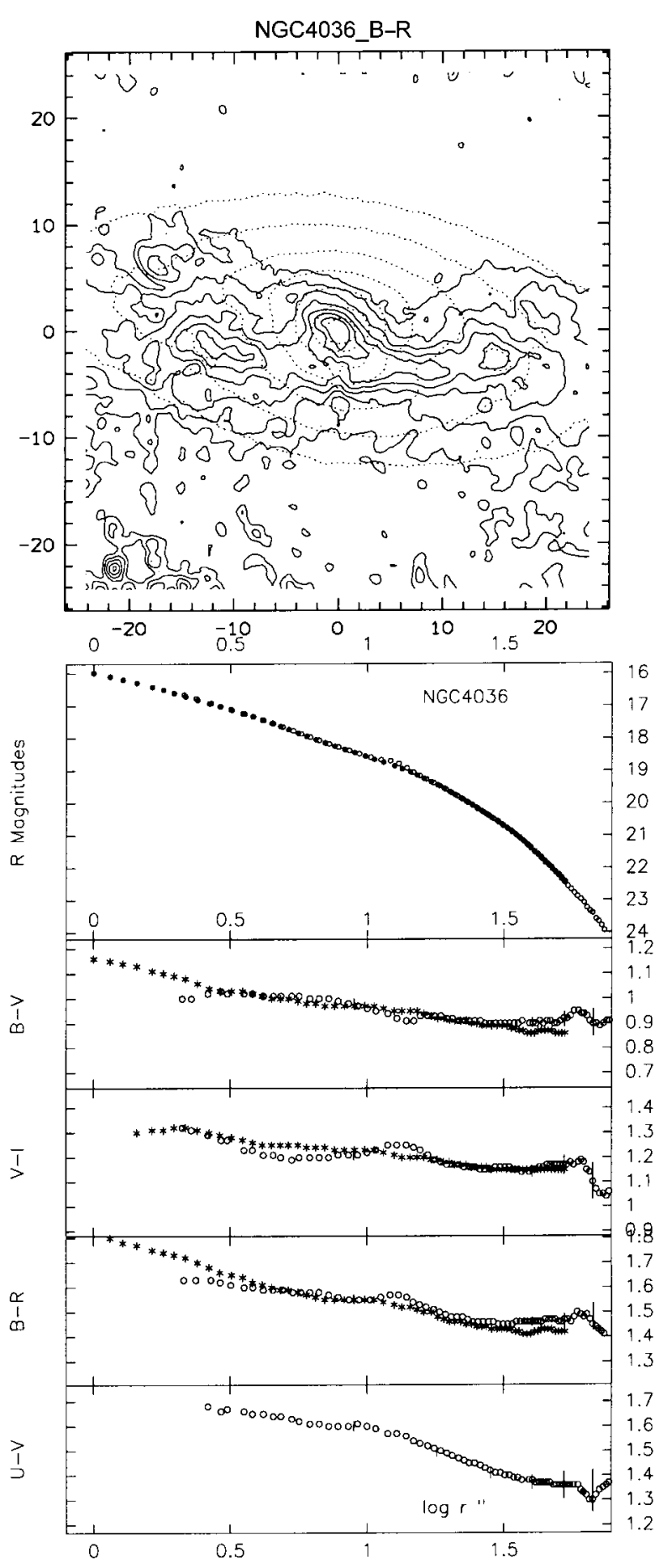

Fig. A6. NGC 4036: $B-R$ isochromes and $B$ isophotes from TBL frames. Isochromes $1.50-1.80(0.05)$. Isophotes $18-21$ (0.5). $N$ at the top. This galaxy features a prominent dust pattern nearly parallel to the majA on the S side. The minA asymmetry extends very far, with the $\mathrm{S}$ side redder and less bright 

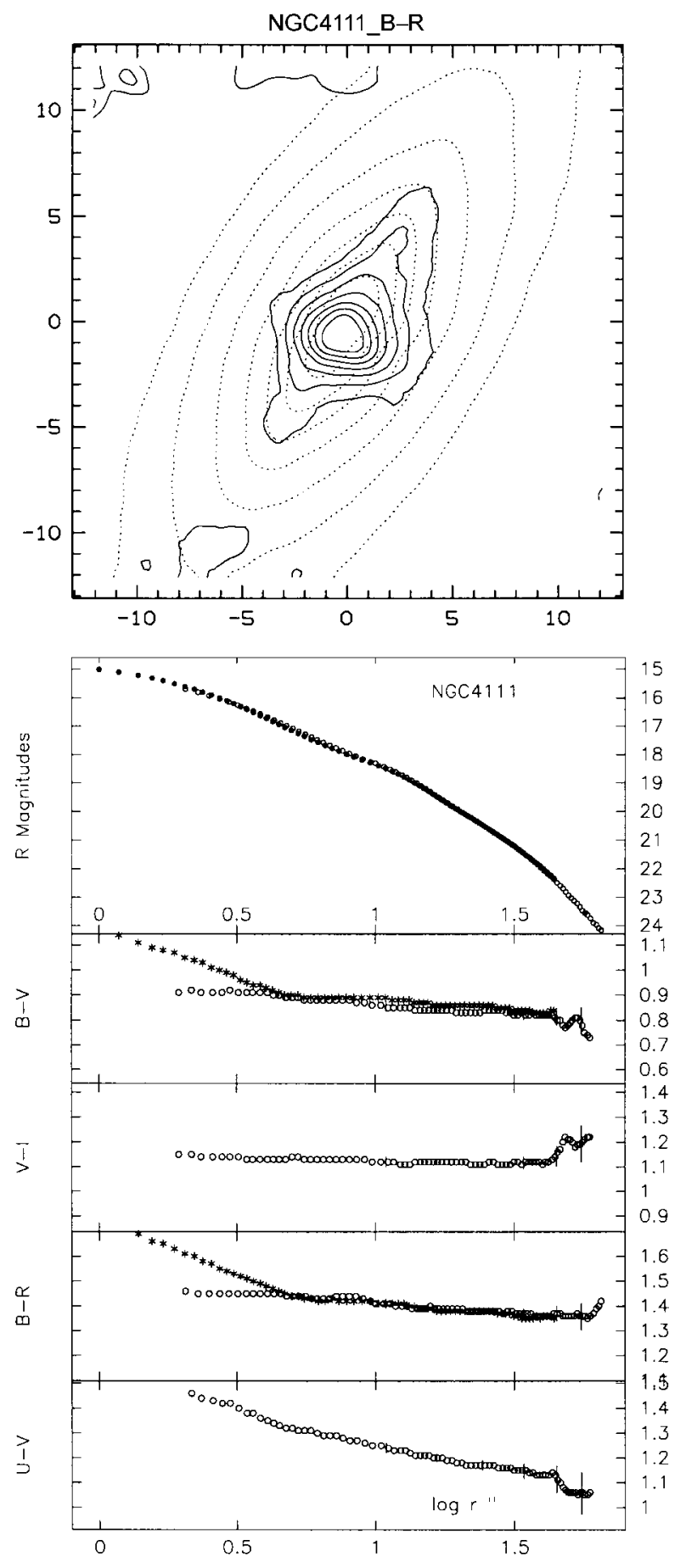

Fig. A7. NGC 4111: $B-R$ isochromes and $B$ isophotes from TBL frames. Isochromes $1.40-1.70(0.05)$. Isophotes $17-20$ (0.5). $N$ at the top. This object displays a sharp central red peak, probably due to dust, with extensions along both the majA and the minA. The minA dust extension and the peanut bulge already noted by Tsikoudi (1972), seem to have correlated geometries
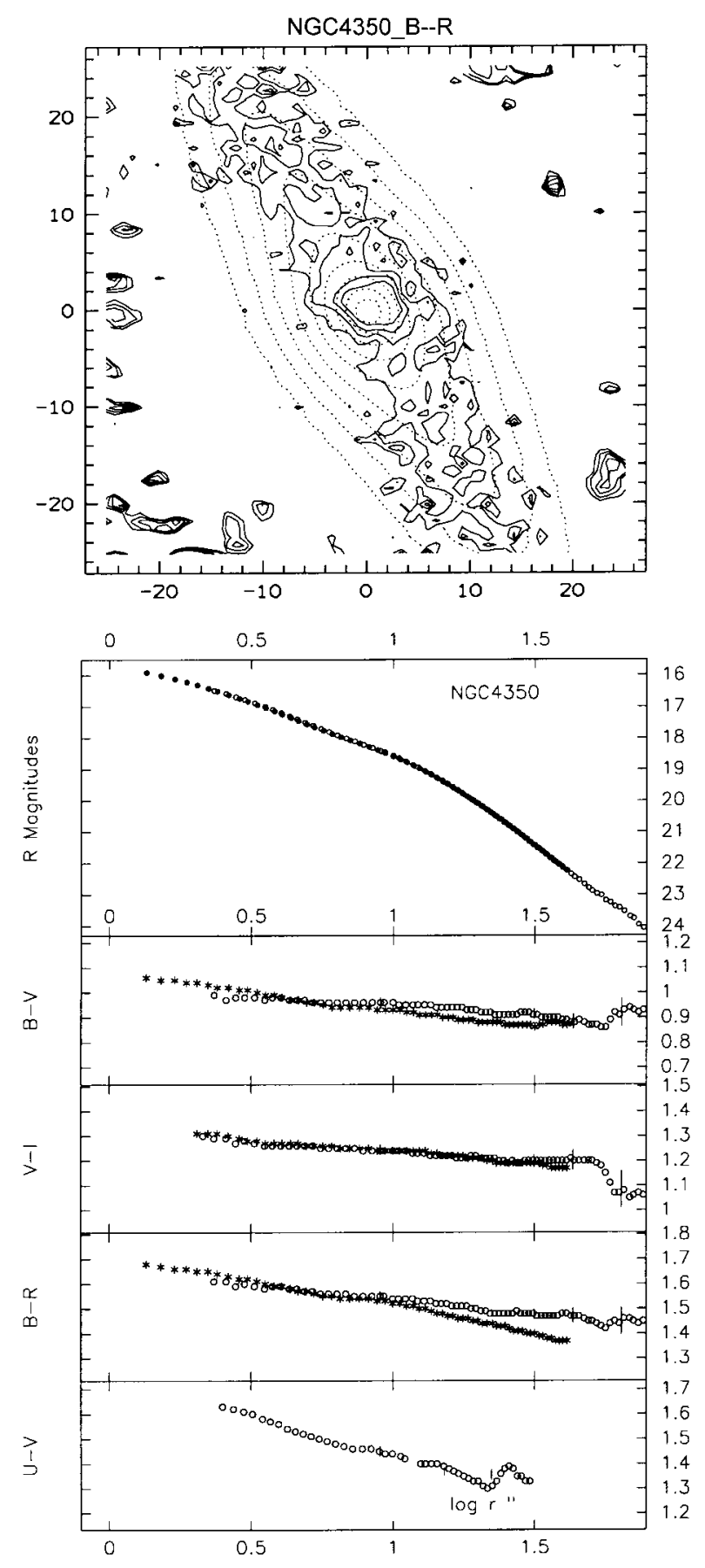

Fig. A8. NGC 4350: $B-R$ isochromes and $B$ isophotes from OHP frames. Isochromes $1.55-1.625$ (0.025). Isophotes $17.5-$ $21.5(0.5) . N$ at the top. The isochromes are flatter and somewhat displaced along the minA, which may be evidence for dust concentration in the disk 


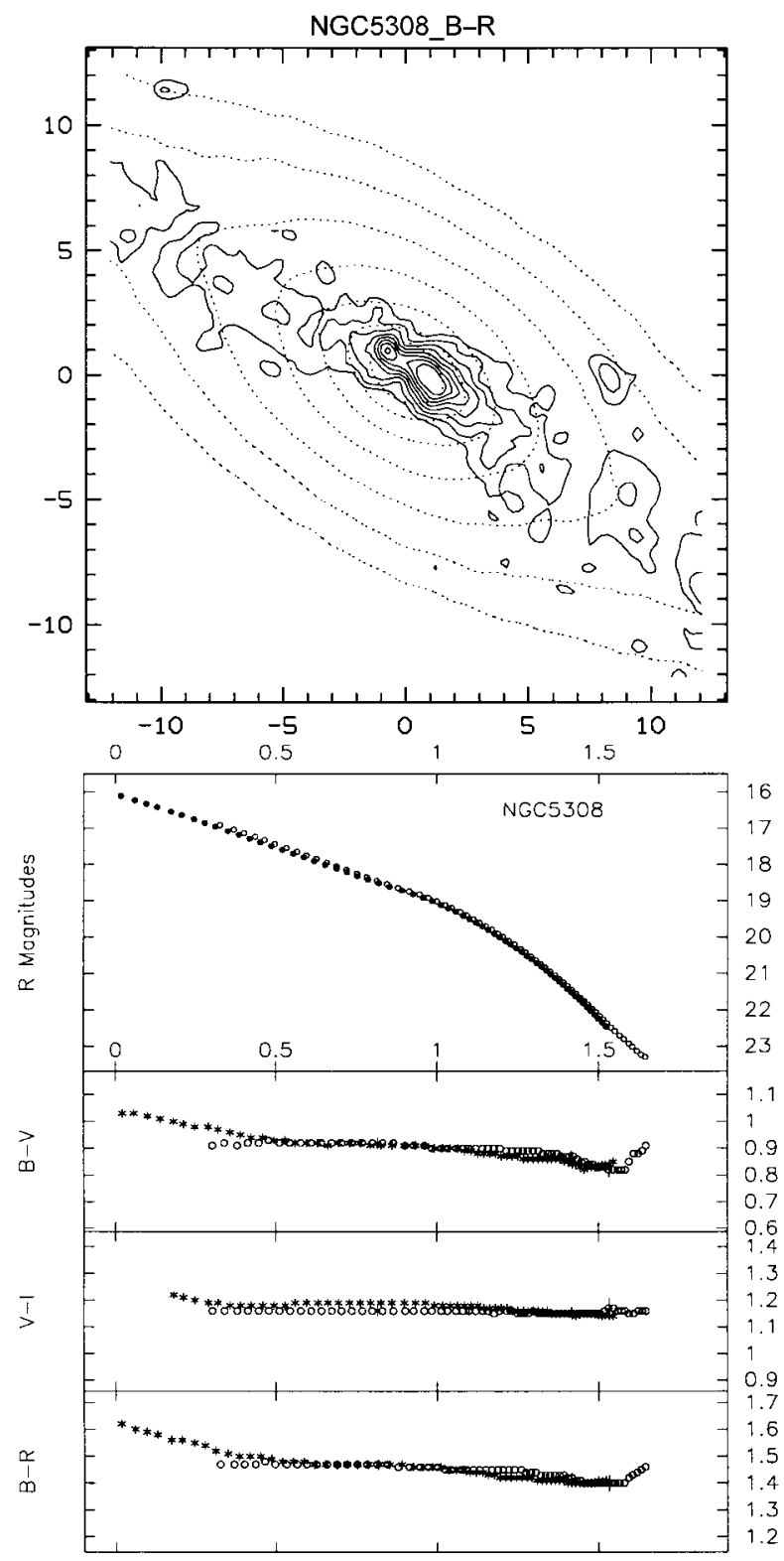

Fig. A9. NGC 5308: $B-R$ isochromes and B isophotes from TBL frames. Isochromes $1.50-1.675$ (0.025. Isophotes $18-21$ (0.5). $N$ at the top. There is a strong reddening of the inner disk, which might be taken as evidence of dust concentration there. The effect is however much smaller in $V-I$ (see also Fig. 3 and Table 10)

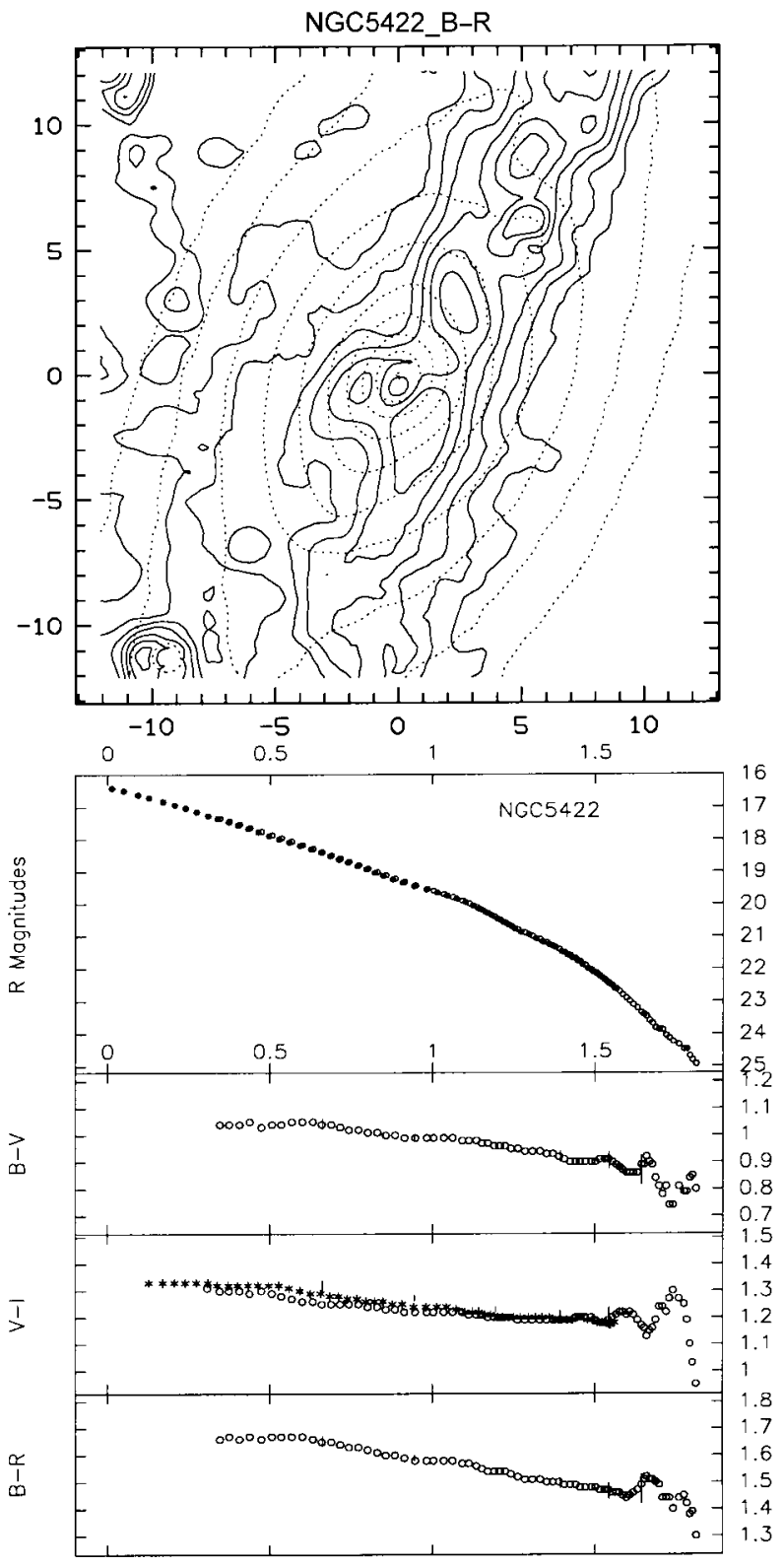

Fig. A10. NGC 5422: $V-I$ isochromes anf $V$ isophotes from TBL frames. Isochromes $1.225-1.375$ (0.025). Isophotes $16.5-$ $21(0.5) . N$ at the top. There is a prominent dust pattern along the majA. This object displays the minA asymmetry in light and colour, the E side being redder and less bright. The colour feature at the SE corner of the field is due to a poorly erased field object 


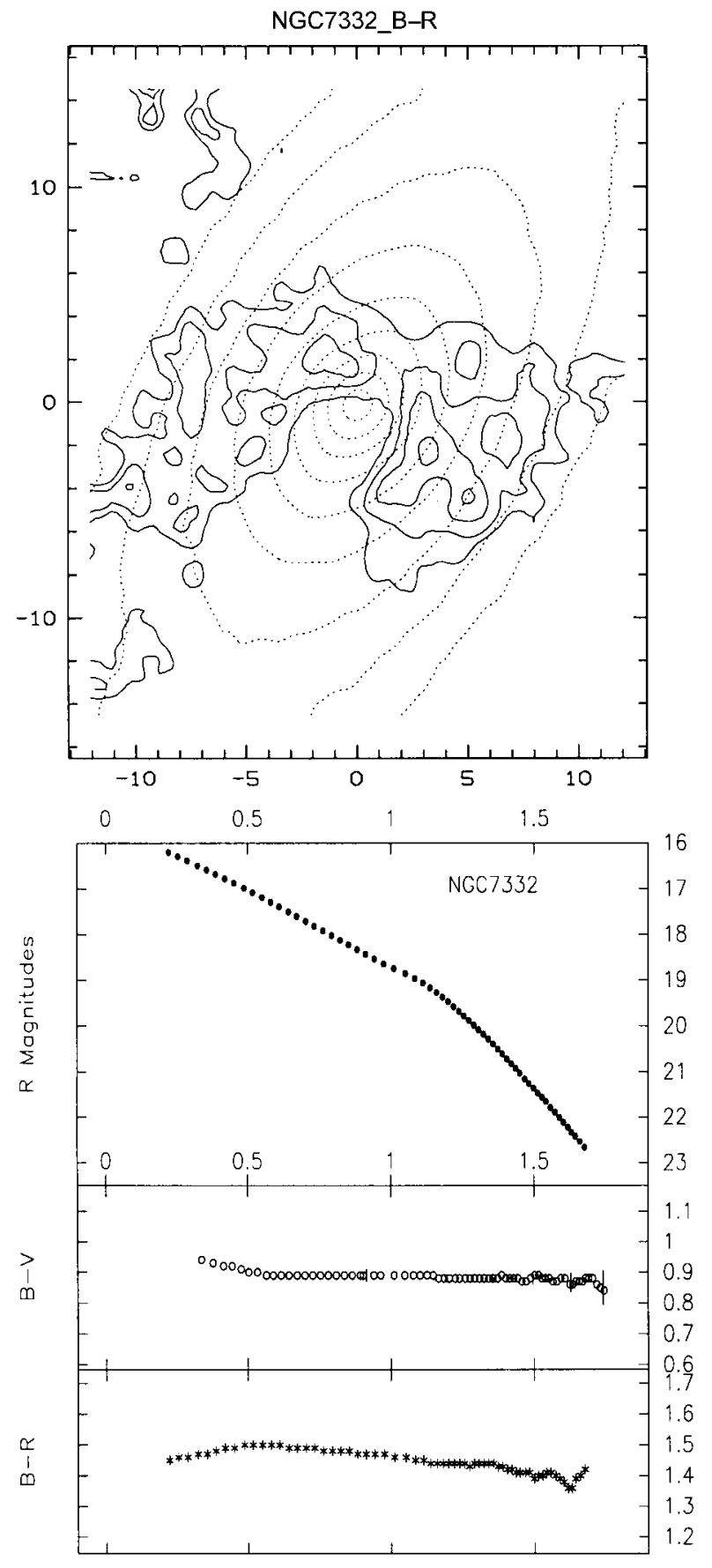

Fig. A11. NGC 7332: $B-R$ isochromes and B isophotes from TBL frames. Isochromes $1.475-1.575$ (0.025). Isophotes $17-21$ (0.5). $N$ at the top. There is an extended dust pattern of modest contrast, extending mostly in the minA direction
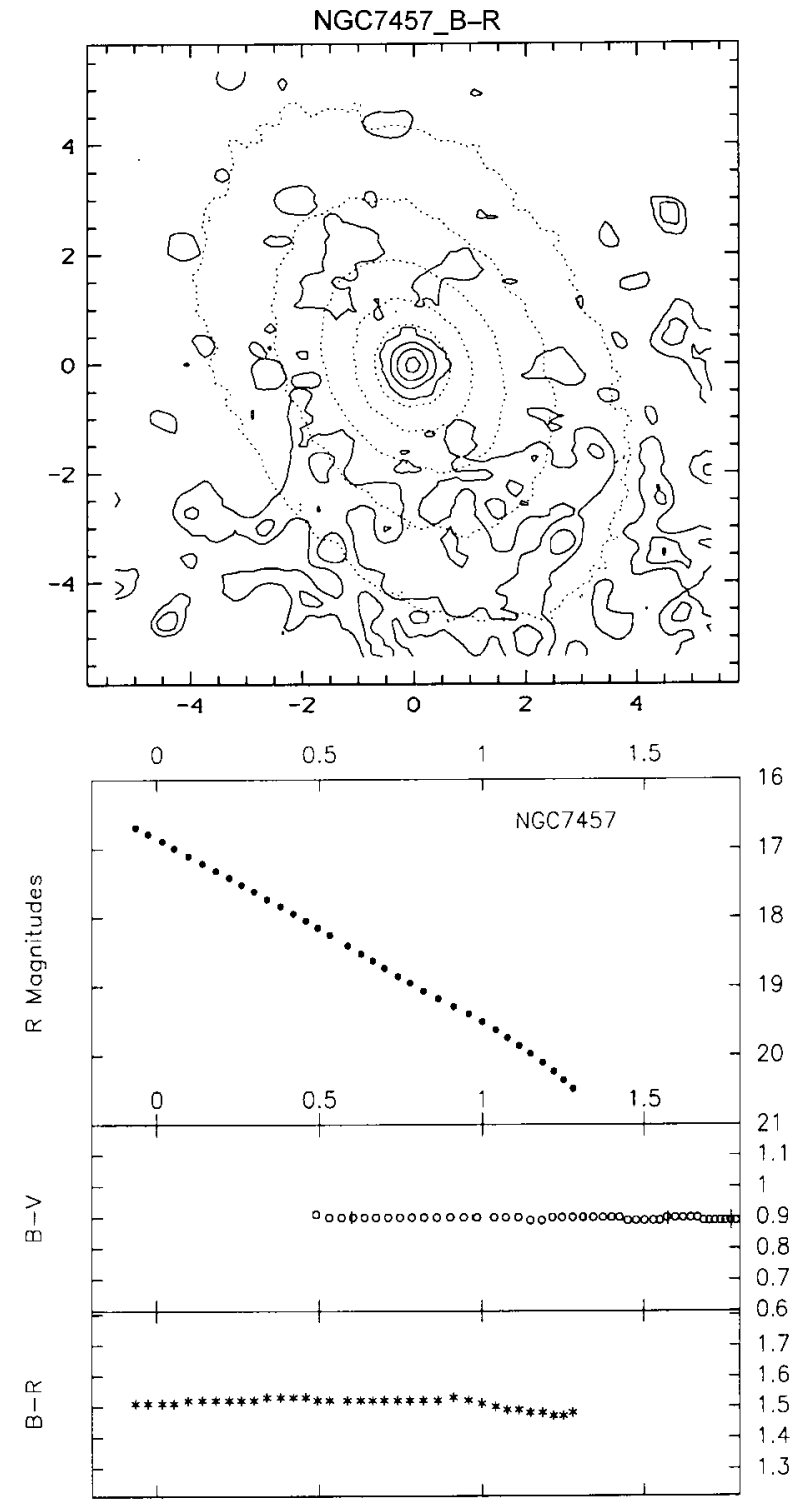

Fig. A12. NGC 7457: $B-R$ isochromes and $B$ isophotes from CFHT frames. Isochromes $1.525-1.60$ (0.025). Isophotes $17-19(0.5) . N$ at 9 o'clock. The colour distribution is quite flat except for a small central red peak 